\title{
Buffet Loading, Dynamic Response and Aerodynamic Control of a Suspension Bridge in a Turbulent Wind
}

\author{
Xiaowei Zhao ${ }^{\mathrm{a}}$, Kevin Gouder ${ }^{\mathrm{b}}$, J. Michael R. Graham ${ }^{\mathrm{c}, \mathrm{d}}$, \\ David J. N. Limebeer ${ }^{\mathrm{e}}$ \\ ${ }^{a} X$. Zhao is with the School of Engineering, University of Warwick, Coventry, CV4 7AL, \\ United Kingdom, e-mail: xiaowei.zhao@warwick.ac.uk \\ ${ }^{b} K$. Gouder is with the Department of Aeronautical Engineering, Imperial College \\ London, London SW7 2AZ, United Kingdom, e-mail: \\ kevin.gouder04@imperial.ac.uk \\ ${ }^{c}$ J.M.R. Graham is with the Department of Aeronautical Engineering, Imperial College \\ London, London SW7 2AZ, United Kingdom, e-mail: m.graham@imperial.ac.uk \\ ${ }^{d}$ Corresponding Author \\ ${ }^{e}$ D.J.N. Limebeer is with the Department of Engineering Science, University of Oxford, \\ Parks Road, Oxford OX1 3JP, United Kingdom, e-mail: \\ david.limebeer@eng.ox.ac.uk
}

\begin{abstract}
This paper describes experiments relating to the buffet response and control of a section of a long-span suspension bridge deck elastically mounted as part of a wind tunnel experiment. The bridge section is subject to grid generated flow turbulence. Two grids are used - one is a standard biplanar grid, while the second is a new design that provides larger turbulence length scales. The buffet response results are compared with admittances calculated using unsteady, three-dimensional, lifting-surface theory that extends standard two-dimensional Sears' theory. The bridge deck heave and pitch responses are predicted with comparisons made with wind tunnel measurements. In order to suppress buffeting, and increase the deck's critical flutter speed, the deck model is fitted with controllable leading- and trailing-edge flaps. Two sets of passive controllers, which use the flap angles as the control inputs, are demonstrated and evaluated for their capability to suppress the buffet response of the deck and increase its critical flutter speed. The first set of controllers sense the deck's position (pitch angle and heave, or pitch angle alone), whilst the second set (which are mechanical controllers) sense the vertical velocity of the flap hinge points. The control system design problem is solved as a mixed $H_{2} / H_{\infty}$ optimisation problem. The wind tunnel exper-
\end{abstract}


iments show that these control systems can reduce considerably the deck's buffet response, whilst simultaneously increasing its critical flutter speed.

Keywords: Long-span bridges, unsteady thin aerofoil theory, buffeting, robust control.

\section{Introduction}

T ONG-span bridges, which may be suspension bridges or cable-stayed bridges, $\amalg$ are known to be sensitive to wind-induced influences. The iconic Tacoma Narrows incident, Billah and Scanlan (1991), is the best known case of a windinduced disaster. However, a significant number of other bridges have also experienced detrimental wind-induced effects in which the bridge response was of sufficiently large amplitude to cause concern. An important wind induced phenomena is aerodynamic instability that is often, but not always, a classical two-degree of freedom heave-pitch flutter. Another wind-induced phenomenon is aerodynamic buffet, which is produced by turbulence in the incident air stream. These phenomena are given careful consideration in the design of any new bridge.

Buffet loading occurs on all bridges, but usually only generates significantly adverse effects on long span bridges in high winds. Turbulence in the incident wind induces a random unsteady aerodynamic load (buffet), which is dominated by the bridge deck's response to the vertical component of the gust velocity. Buffet loading also results from flow separation occurring around a non-streamlined deck. The resulting aerodynamic forces are inherently unsteady due to vortex shedding, but may also interact strongly with the incident turbulence in the wind. This self-buffeting will not be considered explicitly in this paper except within the context of the aerodynamic control of the wind tunnel model.

Aeroelastic analyses of wind sensitive bridge decks is now standard and is usually carried out by employing a strip theory analysis (for the aerodynamics), since the wavelengths of the relevant spanwise structural modes of the deck are large compared with the aerodynamic chord (bridge deck width). However, the theory is less accurate when predicting buffet loads when the spanwise length scale of the incident turbulence is small. This is especially true for wind tunnel simulations where it is often difficult to generate representative large turbulence length scales.

Many bridge decks are comprised of closed, near trapezoidal sections, 
presenting a streamlined thin structure approximating a negatively cambered flat plate. Unsteady thin aerofoil theory has been shown to be quite accurate in determining the aerodynamic derivatives of these bridge sections. Similarly, Sears' theory, Sears (1941), for convected two-dimensional gusts is often used to predict buffet forces on thin bridge sections due to incident turbulence. The strip theory method and the implications of three-dimensionality are discussed fully in Larose and Mann (1998) for example. Previous work, Graham (1970), has extended Sears' theory to three-dimensional flows by considering oblique sinusoidal gusts. Following this work Jackson et al. (1973) showed that aerodynamic derivatives calculated in this way for the individual Fourier components of homogeneous turbulence could predict quite accurately the admittance for induced lift on a thin aerofoil or plate section in a turbulent flow where two dimensional theories, including strip theory, over-predicted the forces.

The aerodynamic stability of suspended-span bridges tends to degrade as the free span of a bridge increases. This results primarily from a reduction in the torsional stiffness of the bridge structure, but the reduced bending stiffness also reduces the critical wind speed. Extreme spans under current consideration require major design changes to preserve the safety margin between the critical flutter speed and the highest expected wind speeds Brown (2001). These changes include widening the deck and separating it into two roadways, but purely structural solutions usually lead to high costs.

Following the Tacoma Narrows disaster the possibility of compensating for adverse aerodynamic influences has been investigated in the context of many bridges. Initially this was in the form of controlling the airflow over the bridge deck with particular attention paid to the aerodynamic shape of the deck, the porosity of the structure and profiling the deck edges. We call this traditional passivity control, which exploits surface shaping, requires no power input, but has limited efficacy.

More recently the possibility of active control using controllable flaps has been investigated Hansen and Thoft-Christensen (2001); Wilde and Fujino (1996). The concept of using flaps for control is not new; it has been used in the aeronautics industry for flutter suppression and buffet loads alleviation, where flaps respond to the wing's oscillatory motion, as seen, for example in Triplett (1972); Triplett et al. (1973); Roger et al. (1975); Karpel (1982); Borglund and Kuttenkeuler (2002); Burnett et al. (2010). The main advantage of feedback control using controllable flaps relative to traditional structural changes is the possibility of raising significantly the critical speed for flutter 
along with considerable potential cost advantages. Actively controlled flaps can similarly be effective in combating buffet forces induced on a bridge deck by turbulence in the oncoming wind. Frederick et al. (2010) showed that a controlled trailing edge flap could reduce by as much as $80 \%$ the amplitude of the buffet loading on an aerofoil due to a strongly turbulent flow. In this case a short (4\% of chord) moving Gurney flap was used to increase the actuation bandwidth. In the case of a bridge deck the relevant response frequencies are low and bandwidth is not a major problem. However, active control requires a power supply, computers and powered actuators, and high winds are likely to disrupt these facilities.

A recent passive mechanical controller concept, which has been successfully utilised in vehicle suspension, Sharma and Limebeer (2012), and motorcycle steering, Evangelou et al. (2007), might offer a 'best-of-both-worlds' solution. This technique does not require a power supply, but offers the benefits of actively controlled flaps. The controller is a mechanical network consisting of springs, dampers and inerters that offers good performance including such things as fast response, robustness and so on. Our recent work Limebeer et al. (2011) has shown that controlled conventional flaps, hinged at the trailing edge of an aerofoil, or thin bridge deck section, and driven by a mechanical controller, can substantially reduce buffet loads induced by incident unsteady flow and can also be used to raise the critical flutter speed. Because the direction of the incident wind may be uncertain, controllable flaps may have to be fitted on both deck edges. This arrangement means that leading- and trailing-edge flaps will be available for control whatever the wind direction. Mechanical control systems, which are insensitive to wind direction, are proposed in Zhao et al. (2015). Controllable leading edge flaps, which are unusual in aircraft, are shown to be capable of making a significant contribution to buffet load reduction and to raising the flutter speed. This flap arrangement may, however, produce adverse effects. A conventional trailing edge flap, in order to be efficient, will usually have a 'sharp' trailing edge. When the wind direction is reversed such a flap, now on the upwind side, presents a sharp leading edge to the wind that can produce early-onset flow separation.

In Limebeer et al. (2011); Zhao et al. (2015), the controls are the torques applied to the controllable flaps, but it is more convenient to use flap angles as the control inputs for wind tunnel experiments. The controls in Graham et al. (2011a), in which two flaps are used, are flap angles. There is no mechanical controller design in Graham et al. (2011a) or Graham et al. (2011b) and the 
control system is sensitive to wind direction. In Zhao et al. (2011) a simple mechanical flutter controller is designed with the flap angles as control inputs and heave velocity as the sensed feedback signal. Unfortunately, our recent wind tunnel tests show that controllers with heave velocity as the feedback cannot suppress adequately the deck's pitch behaviour — this is because flutter could be a pitch-dominated phenomenon. In this paper we present a mechanical bridge control system with the flap angles as the control inputs, and the vertical velocity of the flap hinge points as feedback; this arrangement is insensitive to wind direction. This control system is tested in the wind tunnel.

The paper has two primary objectives. The first is to conduct buffet response experiments on a bridge deck section in a turbulent air stream. Measured and theoretically predicted responses will be compared and analysed. We will compare buffet admittances obtained from wind tunnel experiments with the admittances calculated from unsteady, three-dimensional, thin aerofoil theory. The bridge deck is mounted elastically in the Honda wind tunnel at Imperial College London with heave and pitch freedoms, while the incident stream turbulence is generated by a newly designed grid that provides large length-scale turbulence. We found that the measured results are about 1.7 times higher at the resonant peak for pitch motions than the theoretically predicted values, with closer agreement obtained for heave motions. We suspect that some self-buffet occurred. The second aim of this paper is to conduct buffet control experiments using controllable leading- and trailing-edge flaps. These flaps were mounted on the deck model and suspended elastically in wind tunnel facilities. The control inputs are the leading- and/or trailingedge flap angles. Two controllers were developed: the first senses the deck position, while the second produces flap angle deviations that are responsive to the vertical velocity of the flap hinge points. The wind tunnel tests show that the controllers can improve the critical wind speed for flutter, while simultaneously suppressing buffeting.

The present paper is divided into five sections. Section 2 is dedicated to the experiment setup. Theoretical predictions of the deck's buffet response are given in Section 3. These predicted responses are compared with measured dynamic response for a bridge deck model in wind tunnel turbulence. We also derive a theoretical expression for the modal response of the bridge deck and measure the spectrum of its heave and pitch responses. Theoretical and experimental results are compared. Control experiments are conducted in Section 4. We begin in Section 4.1 with a summary of the 
structural and aerodynamic models of a single bridge deck section with controllable leading- and trailing-edge flaps proposed in the prior publications Graham et al. (2011a,b), which is based on classical thin aerofoil theory. In Section 4.2 we then propose a configuration for the feedback control system. The control system design optimisation is given in Section 4.3. The theoretical and experimental results with the position feedback control system are given in section 4.4, while the results obtained with a mechanical control system are given in Section 4.5. The conclusions are summarised in Section 5.

\section{Experiment setup}

The deck model utilised in these experiments is made from a rigid carbonfibre-composite with chord $0.82 \mathrm{~m}$, span $2.74 \mathrm{~m}$ and depth $0.1 \mathrm{~m}$. The deck's leading and trailing edges were both faired using low-density styrofoam, as advised by BMT Fluid Mechanics, prior to the installation of control surfaces (flaps). The total chord of the deck, including the fairing and the flaps, was $1.09 \mathrm{~m}$ with each flap chord $12 \%$ of the total deck chord.

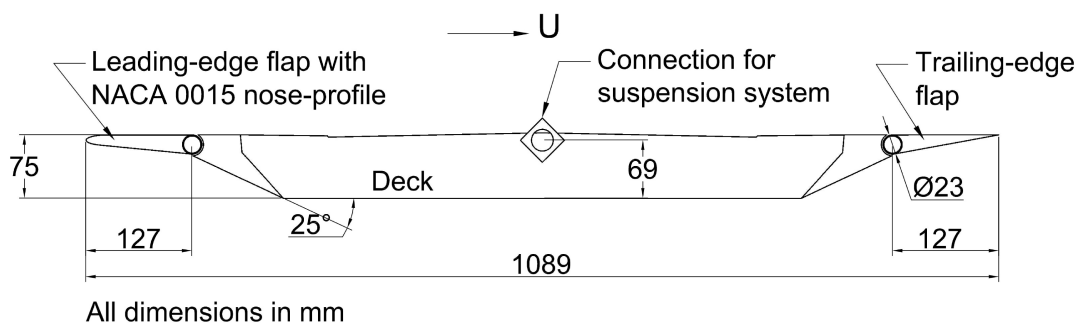

Figure 1: The 1:50 model deck with dimensions.

Both flaps were designed to be triangular in section. However, because separation was found to occur at the sharp leading edge for the upstream flap, when the deck section was responding in heave and pitch there was concern that this was contributing additional buffet loading. While it was found that retaining the sharp leading edge did not appear to reduce control performance, for the purposes of assessing the accuracy of the linear theory in predicting response, in the present tests, a degree of rounding following a NACA0015 profile was applied to the leading edge of this flap only giving it a radius of nose curvature of $3 \mathrm{~mm}$. This is an area where further work will be needed, since in many cases where extreme winds are possible from either 
side of the deck neither flap would permanently operate in the leading edge position and a rounded trailing edge has reduced effectiveness. Each of the flaps was mounted on an aluminium alloy tube and connected to a spur gear 1:5 reduction gearbox driven by a Nanotec ST8918S4508 NEMA34 stepper motor capable of developing a torque of $3 \mathrm{Nm}$. The motor was controlled using a Nanotec SMCI47-S stepper motor positioning controller. The positioning controller was configured using a serial communications link with a desktop computer. The motor was run in the analogue positioning mode, where the motor position was proportional to an analogue voltage input to the SMCI47-S. The SMCI47-S ran an internal (hardware) PID controller that ensured that the motor shaft position tracked the analogue position demand reference. The PID controller's parameters were set prior to the experiment by running a PID parameter wizard on the SMCI47-S. Shaft positional feedback was attained through a WEDS5541-B shaft encoder, which has 1000 counts per revolution, but which was run in quadrature mode thereby quadrupling its position accuracy.

The deck was suspended in BMT's No. 5 tunnel in Teddington, UK. The drive motors and gear boxes at the ends of the deck were outside the tunnel; see Figure 2. In the case of initial experiments run in the Honda wind tunnel at Imperial College, false walls were utilised because the tunnel test-section is wider than the deck. In this case the motors and gearboxes were situated between the false walls and the tunnel walls. The suspension system, shown in Figure 2, comprises an H-assembly of springs that allowed the deck to have torsional and heave freedoms. These springs were tuned to replicate 'real' bridge structure characteristics. Spanwise and streamwise motions were constrained with drag wires. Roll was unconstrained, but the asymmetric aerodynamic forces at the roll frequency were negligible and so this mode was not excited. The bridge deck model's main parameters, such as its reduced frequency $k_{1}=\frac{\omega b}{U}$ (where $b$ is the deck semi-chord, $\omega$ is the system frequency, $U$ is the freestream velocity) and the mass ratio $\frac{M_{h}}{\rho A_{x}}$ (where $M_{h}$ is the deck mass per unit length, $\rho$ is the air density, $A_{x}$ is the deck cross-sectional area) were set to correspond to a generic suspension bridge.

The heave frequency was set using the eight springs forming the two $\mathrm{H}$ assemblies; in our case each of the springs had a stiffness of $1160 \mathrm{~N}$. With the heave frequency set, the frequency ratio could be set by adjusting the torsional frequency using the adjustable anchors. A final frequency ratio $\omega_{p} / \omega_{h}=1.54$ was used. Subscripts $p$ and $h$ are used to denote pitch and 


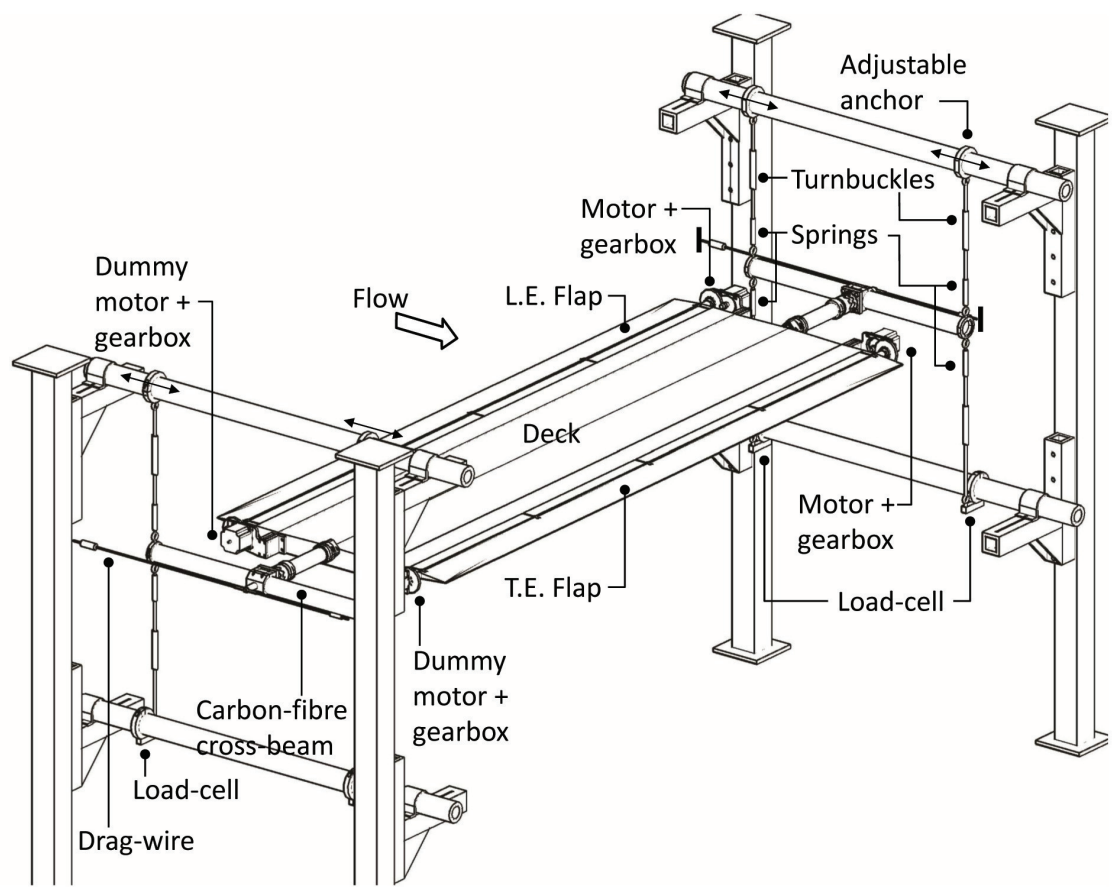

Figure 2: Schematic of the experimental setup of the bridge deck with leading- and trailingedge flaps. The tunnel walls (or false walls) have been omitted for clarity.

heave modes. The deck was balanced and so had no static moment. The drag wires attachment points were selected to minimise the introduction of damping effects. The final values of the structural damping ratios were $\zeta_{h}=0.0057$ and $\zeta_{p}=0.0033$, or in log-dec format, $\delta_{h}=0.036$ and $\delta_{p}=0.021$. The critical flutter speed of the plain deck was found from the wind tunnel tests of the uncontrolled deck to be $U_{c}=17.5 \mathrm{~m} / \mathrm{s}$.

The lower springs in each $\mathrm{H}$-assembly leg were connected to load-cells MIL-LB-8000 driven by a full-bridge ohmic Fylde amplifier. The bridge deck's heave and pitch position can be computed from reaction load measurements and the stiffness of the suspension springs. The bridge deck's position data were acquired on analogue input channels, the motors' shaft position was recorded on digital counter channels and the motors' position demand on the analogue outputs of an NI PCI-6259 data acquisition card.

A turbulence generating bi-planar grid with horizontal and vertical members of cross-section $68 \mathrm{~mm}$ in height $\times 32 \mathrm{~mm}$ in depth was installed up- 
stream of the start of the test section (i.e. towards the end of the contraction). The grid spacing (member centreline to member centreline) was $300 \mathrm{~mm}$, or an opening of $232 \mathrm{~mm} \times 232 \mathrm{~mm}$. This gives a blockage area of $39.7 \%$. Figure 3 shows a photograph of the grid. At the streamwise location of the bridge, this grid produced a vertical velocity turbulence intensity $\sqrt{\overline{u_{3}^{2}}} / U$ of 0.065 and a spanwise length scale of vertical velocity $L_{u_{3}}\left(x_{2}\right) / 2 b$ of 0.05. A new design of grid capable of generating larger length-scales was also used. Again, at the streamwise location of the bridge, this grid produced spanwise lengthscales of $L_{u_{1}}\left(x_{2}\right) / 2 b$ of $0.12-0.18$ and $L_{u_{3}}\left(x_{2}\right) / 2 b$ of 0.08 and (non-uniform across the span) intensities of $\sqrt{\overline{u_{1}^{2}}} / U$ of $0.12-0.15$ and $\sqrt{\overline{u_{3}^{2}}} / U$ of $0.09-0.13$. It had a blockage ratio of $32 \%$.

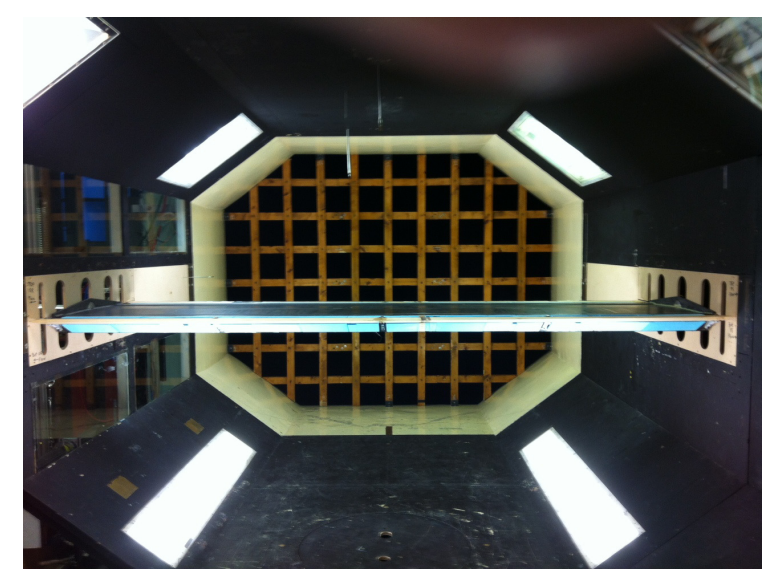

Figure 3: Grid in the BMT wind tunnel.

The cross-wire probe used here was a Dantec 55P61 that was mounted in a standard cross-wire probe holder and driven by a Dantec miniCTA hot-wire anemometer. This probe was calibrated against the wind-tunnel Pitot-static probe - connected to a Betz manometer - at various wind velocities. A reference location was selected for drift checks on the hot-wire probe. At a set wind-speed the probe was taken to this location approximately every hour and the registered mean velocity was compared to the mean velocity measured at the same location after the calibration. A drift in mean velocity of more than $2 \%$ was set as the indicator for the requirement of a recalibration. In order to compute the spanwise length scale of the turbulent vertical velocity component $u_{3}$, the bridge deck was temporarily removed from the 
wind tunnel. The mean and r.m.s values of $u_{1}$ and $u_{3}$ (the turbulent velocity components in the chordwise and normal directions) were recorded at the streamwise location coincident with the mid-chord location of the bridge model. By utilising two cross-wire probes, keeping one probe fixed at tunnel half-height, and moving the other relative to it in the spanwise direction, the spanwise length scale of the vertical velocity $u_{3}$ was measured.

The physical parameters of the deck are summarised in Table 1, which will be used in Section 4.1.

\begin{tabular}{||c||c||}
\hline Parameters & Values \\
\hline$b$ & $0.545 \mathrm{~m}$ \\
\hline$M_{h}$ & $18.9 \mathrm{~kg}$ \\
\hline$J_{p}$ & $1.8 \mathrm{~kg} \mathrm{~m}{ }^{2}$ \\
\hline$\omega_{p}$ & $20.74 \mathrm{rad} / \mathrm{s}$ \\
\hline$\omega_{h}$ & $13.45 \mathrm{rad} / \mathrm{s}$ \\
\hline$\zeta_{p}$ & 0.0033 \\
\hline$\zeta_{h}$ & 0.0057 \\
\hline$\rho$ & $1.23 \mathrm{~kg} / \mathrm{m}^{3}$ \\
\hline
\end{tabular}

Table 1: Physical parameters of the deck. Note that $b$ includes the flap chords.

\section{Theoretical Prediction of Buffet Response}

In this section we make theoretical predictions of the deck's dynamic buffet response when the length-scale

$$
L_{x}=\int_{0}^{\infty} \frac{\overline{u_{1}\left(x_{1}\right) u_{1}\left(x_{1}+x_{1}^{\prime}\right)}}{\overline{u_{1}^{2}\left(x_{1}\right)}} d x_{1}^{\prime} .
$$

of the turbulence necessitates three-dimensional aerodynamics to be considered. Here and in the following an overbar indicates a time average and bold type is used for tensor quantities. The coordinate directions in the chordwise, spanwise and normal (to the deck) directions are given by $x_{1}, x_{2}, x_{3}$ 
respectively. The mean wind speed is denoted $U$. The turbulent velocity components are given by $u_{1}, u_{2}, u_{3}$, with $\kappa_{1}, \kappa_{2}, \kappa_{3}$ the three components of the vector wavenumber, in the corresponding directions.

\subsection{Expressions for Turbulence Spectra}

In the present work we will assume that the von-Karman spectrum, Deaves and Harris (1978), gives a satisfactory representation of the grid generated turbulence used in the wind tunnel tests as well as turbulence in a typical neutral atmospheric boundary layer. Other representations such as the Kaimal spectrum, Kaimal et al. (1972), may be considered. The vonKarman spectrum intended to represent homogeneous, isotropic turbulence with a substantial inertial subrange, described by the triple wave number spectrum function for the turbulent velocity, is:

$$
\Phi_{i j}\left(\kappa_{1}^{*}, \kappa_{2}^{*}, \kappa_{3}^{*}\right)=\frac{55 a^{2} \overline{u^{2}} L_{x}^{3} \kappa^{* 2}}{9 U\left(1+\kappa^{* 2}\right)^{\frac{17}{6}}}\left\{\delta_{i j}-\frac{\kappa^{* 2}-\kappa_{i}^{*} \kappa_{j}^{*}}{\kappa^{* 2}}\right\}
$$

where

$$
\kappa^{*}=a L_{x} \sqrt{\kappa_{1}^{2}+\kappa_{2}^{2}+\kappa_{3}^{2}}
$$

and $a$ is the ratio of Gamma functions

$$
a=\frac{\Gamma\left(\frac{1}{3}\right)}{\Gamma\left(\frac{1}{2}\right) \Gamma\left(\frac{5}{6}\right)} .
$$

Turbulence in the natural wind is anisotropic and it is therefore usual to replace $\overline{u^{2}}$ in (2) by the relevant turbulence components.

The correspondiong double wave number spectrum function, which represents the stochastic distribution of the vertical velocity component $u_{3}$ of the turbulence in the horizontal plane of the bridge deck is

$$
\begin{aligned}
\Phi_{33}\left(\kappa_{1}^{*}, \kappa_{2}^{*}\right) & =\int_{0}^{\infty} \Phi_{33}\left(\kappa_{1}, \kappa_{2}, \kappa_{3}\right) d \kappa_{3} \\
& =\frac{16 \overline{u_{3}^{2}} a^{2} L_{x}^{2}}{9 U}\left\{\frac{\kappa_{1}^{* 2}+\kappa_{2}^{* 2}}{\left(1+\kappa_{1}^{* 2}+\kappa_{2}^{* 2}\right)^{\frac{7}{3}}}\right\}
\end{aligned}
$$

and the single wave-number (frequency) spectrum of $u_{3}$ is

$$
\begin{aligned}
\phi_{33}\left(\kappa_{1}^{*}\right) & =\int_{0}^{\infty} \Phi_{33}\left(\kappa_{1}, \kappa_{2}\right) d \kappa_{2} \\
& =\frac{2 \overline{u_{3}^{2}} L_{x}}{3 U}\left\{\frac{3+8 \kappa_{1}^{* 2}}{\left(1+\kappa_{1}^{* 2}\right)^{\frac{11}{6}}}\right\} .
\end{aligned}
$$


The admittance of lift is the ratio of the single wave-number (frequency) 'output' spectrum of lift on the element of bridge deck under test, $\phi_{L L}\left(\kappa_{1}^{*}\right)$, to the 'input' spectrum of vertical velocity of the incident turbulence $\phi_{33}\left(\kappa_{1}^{*}\right)$ at corresponding values of $\kappa_{1}^{*}$. This depends on the ratio:

$$
\begin{aligned}
\Psi\left(\kappa_{1}^{*}, \kappa_{2}^{*}\right) & =\frac{\Phi_{33}\left(\kappa_{1}^{*}, \kappa_{2}^{*}\right)}{\phi_{33}\left(\kappa_{1}^{*}\right)} \\
& =\frac{8 a^{2} L_{x}\left(\kappa_{1}^{* 2}+\kappa_{2}^{* 2}\right)\left(1+\kappa_{1}^{* 2}\right)^{\frac{11}{6}}}{3\left(3+8 \kappa_{1}^{* 2}\right)\left(1+\kappa_{1}^{* 2}+\kappa_{2}^{* 2}\right)^{\frac{7}{3}}} .
\end{aligned}
$$

In order to carry out the required spanwise wave-number-structural mode integration to obtain the modal response of the bridge deck to turbulence efficiently, it is convenient to represent the factors in $\Psi$, which depend on the spanwise wavenumber $\kappa_{2}$, by a rational approximation, Limebeer et al. (2011).

\subsection{Bridge Response}

The bridge deck heave and pitch response to turbulence is dominated by the sensitivity to the vertical velocity component. The prediction of this response, when linear theory can be assumed, is carried out by considering each wavenumber component $\hat{u}_{3} e^{i\left(\omega t-\kappa_{1} x_{1}-\kappa_{2} x_{2}\right)}$ of the Fourier transform of the vertical velocity of the turbulence in the $x_{3}=0$ plane of the bridge deck independently. The turbulence is treated as convected 'frozen' by the local mean wind velocity $U$, therefore $\kappa_{1}=\omega / U$. Unsteady thin aerofoil theory has been widely used for calculating the unsteady loading on the more streamlined types of bridge deck of thickness to chord ratio less than $20 \%$, Ostenfeld and Larsen (1992), Selberg and Hjorth-Hansen (1976), Dyrbye and Hansen (1995), Larose and Livesey (1997). The effective angles of attack induced by turbulence are generally small, particularly for the intensities and length scales of wind tunnel turbulence. Separations on the deck which were observed from pressure distribution data to be limited in size are unlikely to be enhanced and more likely to be somewhat suppressed by this level of turbulence. The major effect of separation apart from possible separation associated with the sharp leading edge flap discussed later, is due to the separation over the trailing edge of the bridge deck. This reduces the effectiveness of the assumed Kutta-Joukowski condition fixing circulation and also the effectiveness of the trailing edge flap. An indication of the accuracy of thin aerofoil theory for the forces on the present model bridge deck can 
be seen in the measured value of lift coefficient gradient against angle of attack shown in Figure 12 compared with $2 \pi$ and in the measured aerodynamic derivatives in Figure 21 in Gouder et al. (2015). On the other hand, the reduced effectiveness of the trailing edge flap is shown in Figure 23 in Gouder et al. (2015). Therefore, assuming thin aerofoil theory, the lift force exerted on each section of a bridge deck, of spanwise length $\delta x_{2}$, by a locally correlated $2-\mathrm{D}$ sinusoidal velocity component $\hat{u}_{3} e^{i\left(\omega t-\kappa_{1} x_{1}\right)}$ is:

$$
L(t)=2 \pi \rho U b \delta x_{2} S\left(k_{1}\right) \hat{u}_{3} e^{i \omega t}
$$

where $S\left(k_{1}\right)$ is the Sears function

$$
S\left(k_{1}\right)=\frac{J_{0}\left(k_{1}\right) H_{1}^{(2)}\left(k_{1}\right)-J_{1}\left(k_{1}\right) H_{0}^{(2)}\left(k_{1}\right)}{H_{1}^{(2)}\left(k_{1}\right)+i H_{0}^{(2)}\left(k_{1}\right)} .
$$

The $J_{j}$ 's are Bessel functions, and the $H_{j}^{(2)}$ 's are Hankel functions of the second kind; $k_{1}=\kappa_{1} b$ and $k_{2}=\kappa_{2} b$ are the reduced frequency, and the reduced spanwise wavenumber, respectively, referenced to the semi-chord $b$ following Theodorsen (1934). The function $L(t)$ in (9) is the lift commonly used in strip theory, in which the spanwise variation of $u_{3}$ is applied independently to the loading.

More accurately, the lift induced on the bridge deck section of spanwise length $\delta x_{2}$ by a general Fourier component of the 3-dimensional convected turbulence is:

$$
L\left(x_{2}, t\right)=2 \pi \rho U b \delta x_{2} G\left(k_{1}, k_{2}\right) \hat{u}_{3} e^{i\left(\omega t-\kappa_{2} x_{2}\right)}
$$

where $G\left(k_{1}, k_{2}\right)$ is the 2-wavenumber lift function evaluated numerically in Graham (1970), which may be conveniently written as $G\left(k_{1}, k_{2}\right)=g\left(k_{1}, k_{2}\right) S\left(k_{1}\right)$ with $g\left(k_{1}, 0\right)=1.0$.

An admittance function for the spectrum of lift induced by convected turbulence incident on a finite spanwise length $l$ of bridge deck may now be obtained by integrating the response to each Fourier mode over $l$ and over all spanwise wavenumbers $\kappa_{2}$ weighted by the power density in each wavenumber component of the turbulence. 


\subsection{Modal Response of Deck}

We now consider the excitation of a spanwise structural heave mode $h=$ $\hat{h} \lambda\left(x_{2}\right) e^{i \omega t}$ of a bridge $-l / 2<x_{2}<l / 2$. The modal excitation force is:

$$
\begin{aligned}
F_{L} & =\int_{-\frac{l}{2}}^{\frac{l}{2}} \lambda\left(x_{2}\right) d L \\
& =2 \pi \rho U b \int_{-\frac{l}{2}}^{\frac{l}{2}} \lambda\left(x_{2}\right) g\left(k_{1}, k_{2}\right) S\left(k_{1}\right) \hat{u}_{3} e^{i\left(\omega t-\kappa_{2} x_{2}\right)} d x_{2} .
\end{aligned}
$$

Assuming, for simplicity, that the bridge can be approximated by an undamped elastic beam of uniform mass per unit length $M_{h}$, and stiffness $\sigma_{h}$, the general modal displacement $h\left(x_{2}, t\right)=\lambda\left(x_{2}\right) e^{i \omega t}$ satisfies:

$$
\sigma_{h} \frac{\partial^{4} h}{\partial y^{4}}+M_{h} \frac{\partial^{2} h}{\partial t^{2}}=\hat{F}_{L}\left(x_{2}\right) e^{i \omega t}
$$

where $F_{L}\left(x_{2}, t\right)$ is distributed aerodynamic lift. Assuming pin-jointed end conditions the modal solution is:

$$
h\left(x_{2}, t\right)=\sum_{n}\left\{\hat{h}_{n} \cos \frac{(2 n-1) \pi x_{2}}{l} e^{i \omega_{n} t}\right\},
$$

where the natural frequency $\omega_{n}$ of the $n^{\text {th }}$ mode satisfies

$$
\omega_{n}^{2}=\frac{\sigma}{M}\left\{\frac{(2 n-1) \pi}{l}\right\}^{4} .
$$

Substituting this into (13), multiplying by $\cos \frac{(2 m-1) \pi x_{2}}{l}$ and integrating with respect to $x_{2}$ gives:

$$
\hat{h}_{m}=\frac{2}{M_{h}\left\{\omega_{m}^{2}-\omega^{2}\right\} l} \int_{-l / 2}^{l / 2} \hat{F}_{L}\left(x_{2}\right) \cos \left\{\frac{(2 m-1) \pi x_{2}}{l}\right\} d x_{2} .
$$

For general suspension bridge mode shapes an orthogonal set of $\lambda_{m}\left(x_{2}\right)$ must be determined by structural analysis. In general:

$$
\hat{h}_{m}=\frac{\int_{-l / 2}^{l / 2} \hat{F}_{L}\left(x_{2}\right) \lambda_{m}\left(x_{2}\right) d x_{2}}{M_{h}\left(\omega_{m}^{2}-\omega^{2}\right) \int_{-l / 2}^{l / 2} \lambda_{m}^{2}\left(x_{2}\right) d x_{2}} .
$$


The modal response in pitch may be calculated similarly.

The present wind tunnel experiment, in which a rigid model deck is mounted on springs, has heave and pitch freedoms, with mode shapes satisfying:

$$
\lambda\left(x_{2}\right)=1,\left|x_{2}\right| \leq l / 2 ; \lambda\left(x_{2}\right)=0,\left|x_{2}\right|>l / 2 .
$$

Integrating the $e^{-i \kappa_{2} x_{2}}$ factor in $F_{L}\left(x_{2}\right)$ over the spanwise extent of the model bridge deck gives

$$
F_{L}=2 \pi \rho U b l g\left(k_{1}, k_{2}\right) S\left(k_{1}\right)\left\{\frac{\sin \left(k_{2} A R\right)}{\left(k_{2} A R\right)}\right\} \hat{u}_{3} e^{i \omega t}
$$

where AR is the aspect ratio $l /(2 b)$ of the spanwise length $l$ of deck. The corresponding one-dimensional spectra are obtained by multiplying $F_{L}$ by its complex conjugate and integrating over all spanwise wavenumbers $\kappa_{2}$. Therefore, converting lift force to lift coefficient $C_{L}=\frac{F_{L}}{\rho U^{2} b l}$, the power spectral density of the lift coefficient is:

$$
\begin{aligned}
\phi_{C_{L} C_{L}}\left(k_{1}\right) & =2 \frac{(2 \pi)^{2}}{U^{2}}\left|S\left(k_{1}\right)\right|^{2} \cdots \\
& \int_{0}^{\infty}\left|g\left(k_{1}, k_{2}\right)\right|^{2}\left\{\frac{\sin \left(k_{2} A R\right)}{\left(k_{2} A R\right)}\right\}^{2} \Phi_{33}\left(\kappa_{1}^{*}, \kappa_{2}^{*}\right) d \kappa_{2} .
\end{aligned}
$$

The lift force admittance function $A_{L}(\omega)$ is defined as:

$$
\left|A_{L}(\omega)\right|^{2}=\frac{U^{2} \phi_{C_{L} C_{L}}\left(k_{1}\right)}{(2 \pi)^{2} \phi_{33}\left(\kappa_{1}^{*}\right)}
$$

hence

$$
\begin{aligned}
& \left|A_{L}(\omega)\right|^{2}=\frac{2}{\pi}\left|S\left(k_{1}\right)\right|^{2} \cdots \\
& \int_{0}^{\infty}\left|g\left(k_{1}, k_{2}\right)\right|^{2}\left\{\frac{\sin \left(k_{2} A R\right)}{\left(k_{2} A R\right)}\right\}^{2} \Psi_{33}\left(\kappa_{1}^{*}, \kappa_{2}^{*}\right) d \kappa_{2} .
\end{aligned}
$$

The integrand in equation (22) consists of two factors that represent the effects of three-dimensionality. The factor $\left\{\frac{\sin \left(k_{2} A R\right)}{\left(k_{2} A R\right)}\right\}^{2} \Psi_{33}\left(\kappa_{1}^{*}, \kappa_{2}^{*}\right)$ derives 
from the lack of correlation between the lift force induced on different spanwise strips of the deck and reflects the lack of correlation in the turbulence velocity $u_{3}$ between these $x_{2}$-stations. The $\left|g\left(k_{1}, k_{2}\right)\right|^{2}$ term expresses the additional effect of three-dimensionality in thin aerofoil theory, which gives the lift forces from the vertical velocity distribution. If, as is usually the case at full scale in natural wind, the length scale of the turbulence is large compared with the width (chord) of the bridge deck, the energy in the turbulence spectrum is concentrated in regions of small $k_{2} ; g\left(k_{1}, k_{2}\right)$ takes values close to 1.0 over most of the integral. Replacing $g$ by 1.0 and integrating with respect to $x_{2}$ gives the strip theory result:

$$
\left|A_{L}(\omega)\right|^{2}=\frac{2}{\pi}\left|S\left(k_{1}\right)\right|^{2} \int_{0}^{\infty}\left\{\frac{\sin \left(k_{2} A R\right)}{\left(k_{2} A R\right)}\right\}^{2} \Psi_{33}\left(\kappa_{1}^{*}, \kappa_{2}^{*}\right) d \kappa_{2} .
$$

Changing the order of the spatial $\left(x_{2}\right)$ and wavenumber $\left(\kappa_{2}\right)$ integrations, this result may be written alternatively as:

$$
\left|A_{L}\left(k_{1}\right)\right|^{2}=\left[\frac{2}{l} \int_{0}^{l} R_{33}\left(\omega, x_{2}\right)\left\{1-\frac{x_{2}}{l}\right\} d x_{2}\right]\left|S\left(k_{1}\right)\right|^{2}
$$

where

$$
R_{33}\left(\omega, x_{2}\right)=\frac{2^{\frac{1}{6}} x_{2}^{* \frac{5}{6}}}{\Gamma\left(\frac{5}{6}\right)}\left\{K_{\frac{5}{6}}\left(x_{2}^{*}\right)-\frac{3 x_{2}^{*}}{3+8 \kappa_{1}^{* 2}} K_{\frac{1}{6}}\left(x_{2}^{*}\right)\right\}
$$

is the coherence of the turbulent vertical velocity $u_{3}$ and $K_{\nu}$ is a modified, order $\nu$, Bessel function of the second kind. The expression for $R_{33}\left(\omega, x_{2}\right)$ is derivable directly from the von Karman spectrum formula in equation (2) and $x_{2}^{*}$ is the scaled transverse coordinate $\frac{\sqrt{1+\kappa_{1}^{* 2}}}{a L_{x}} x_{2}$.

In turbulence with a small length scale to the bridge deck chord ratio, as is typically the case in wind tunnel tests, the 3-dimensional aerodynamics considerably reduces the unsteady forces induced by turbulence below the predictions of strip theory with 2-dimensional aerodynamics. Figure 4 shows an example of the measured spectrum of lift generated on the model bridge deck by the grid turbulence in a wind tunnel test, which is compared with the full 3-dimensional (non-strip theory) prediction of equation (22). The admittance has been calculated assuming a reduced value of turbulence length scale 
to bridge chord of $L_{x} /(2 b)=0.17$. Jackson et al. (1973) found that the best fit to the measured spanwise cross-spectra of grid turbulence was obtained by applying a factor of 0.75 to the correlation length predicted by assuming isotropy. The same factor has been found to be appropriate here and has been applied to the length scale $L_{x}$ in the admittance calculation. These measurements were made with the model bridge deck rigidly supported on stiff load cells. The prominent peaks in the measured spectrum, which are beyond the frequency range of main response of the flexibly mounted bridge, are due to residual resonances in the supposedly stiff system.

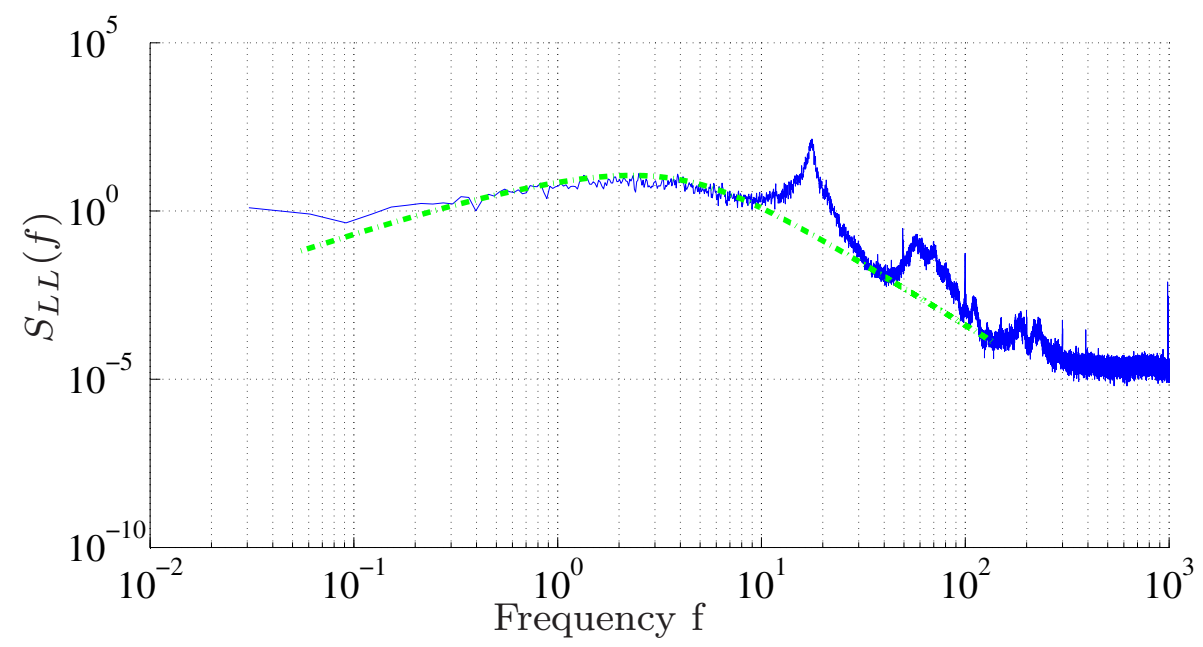

Figure 4: Measured spectrum (solid blue) of the lift generated on the model bridge deck by grid turbulence in a wind tunnel test with a length scale ratio of $L_{x} /(2 b)=0.17$, and its theoretical prediction (dash-dot green). The lift spectrum $S_{L L}(f)$ is in $N^{2} s$ while the frequency $f$ is in $\mathrm{Hz}$.

A similar analysis generates an admittance function $A_{M}\left(k_{1}\right)$ for the pitching moment about the mid-chord. It is worth noting that in two-dimensional flow unsteady thin aerofoil theory predicts that a convected gust of any shape induces the same chordwise load distribution over a flat plate section as is induced by steady incidence in uniform flow Graham (1970). It is merely the amplitude that changes with time as the gust passes. Hence the midchord pitching moment coefficient $C_{M}=\frac{\text { Moment }}{\rho U^{2} b^{2}}$, in Theodorsen's notation, will always be equal to exactly half the lift coefficient $C_{L}$. But the effect of three-dimensionality in the flow moves the centre of lift further towards the 
leading edge and hence increases the ratio of $C_{M}$ to $C_{L}$.

The rigid restraints on the bridge deck support system were then removed and the deck made free to respond in heave and pitch on its spring supports. Its responses in the grid generated turbulence were measured, and the resulting heave and pitch spectra are shown in Figure 5, which also shows the corresponding theoretical predictions. It is clear from Figure 5 that the general shape of the response spectra is accurately predicted, but the measured PSD of pitch exceeds the theoretical prediction at the resonant peak by a factor of approximately 3 (and therefore by about 1.7 in pitch amplitude), while heave shows better agreement throughout. We believe that this difference is due to self-buffeting. This is likely to generate an additional self-buffet input, particularly in pitch. Differences at the low-frequency ends of the spectra are probably due to discrepancies between the assumed von-Karman model spectrum for the vertical velocity and the actual turbulence spectrum. At frequencies above $10 \mathrm{~Hz}$, which are beyond the main response region, the measured spectra show additional system resonance peaks. The spring support system also allowed a third degree-of-freedom (roll) to occur, but it was observed that the roll response was extremely small. The roll mode was not measured or analysed beyond noting that the small $L_{x} /$ span ratio would produce a small roll mode response prediction. The measurements were all carried out at wind speeds below the critical value for classical flutter, since

for full scale bridges the latter is designed to be greater than any wind speed likely to be encountered.

\section{Buffet control}

\subsection{Dynamic Model}

Structural and aerodynamic models of the bridge section, with leadingand trailing-edge controllable flaps, are required for controller design studies Graham et al. (2011a,b).

In the structural model, the heave and pitch dynamics of the bridge section are described by:

$$
\begin{gathered}
M_{h} \ddot{h}+2 M_{h} \omega_{h} \zeta_{h} \dot{h}+K_{h} h=L, \\
J_{p} \ddot{\alpha}+2 J_{p} \omega_{p} \zeta_{p} \dot{\alpha}+K_{p} \alpha=M,
\end{gathered}
$$

where $h$ and $\alpha$ are the deck's heave and pitch angle as illustrated in Fig. 6. The aerodynamic lift force $L$ is positive downwards; the aerodynamic moment around $O$, as shown in Fig. 6 , is positive clockwise; $M_{h}$ and $J_{p}$ are the 


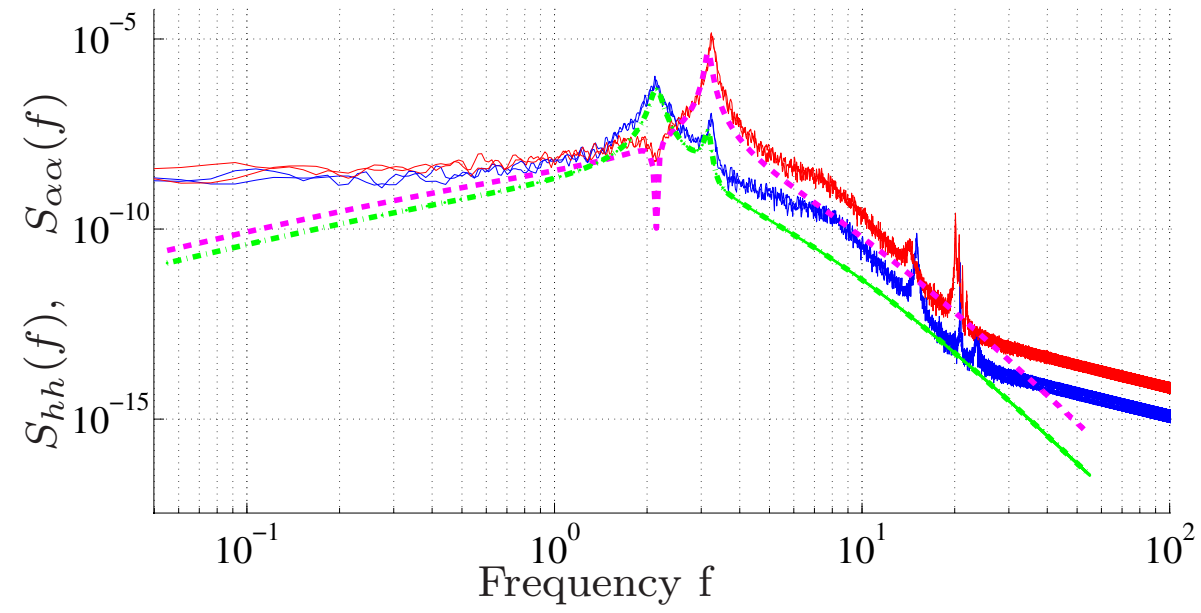

Figure 5: Measured spectra of the heave (solid blue) and pitch (solid red) responses of the model bridge deck in wind tunnel turbulence. The theoretical spectra of the heave and pitch responses in von-Karman spectrum turbulence are shown as (dash-dot green) and (dashed magenta) respectively. The heave and pitch response spectra $S_{h h}(f)$ and $S_{\alpha \alpha}(f)$ are in $m^{2} s$ and $s$ respectively, while the frequency is in $\mathrm{Hz}$.

mass and the torsional mass moment of inertia, per unit length, respectively; $\omega_{h}$ and $\omega_{p}$ are the undamped natural frequencies of the heave mode and pitch mode; $\zeta_{h}$ and $\zeta_{p}$ are the structural damping coefficients; $K_{h}=M_{h} \omega_{h}^{2}$ and $K_{p}=J_{p} \omega_{p}^{2}$ are the per unit length heave and torsional deck stiffness respectively.

The aerodynamic model is based on unsteady thin aerofoil theory as was described in the first instance by Theodorsen (1934), which produces the aerodynamic lift $L$ and moment $M$

$$
\begin{gathered}
L=\rho b^{3} \omega^{2}\left\{L_{h} \frac{h}{b}+L_{\alpha} \alpha+L_{\beta_{t}} \beta_{t}+L_{\beta_{l}} \beta_{l}\right\}, \\
M=\rho b^{4} \omega^{2}\left\{M_{h} \frac{h}{b}+M_{\alpha} \alpha+M_{\beta_{t}} \beta_{t}+M_{\beta_{l}} \beta_{l}\right\}
\end{gathered}
$$

where $\beta_{l}$ and $\beta_{t}$ are the leading- and trailing-edge flap angles as illustrated in Fig. 6. The parameters $b$ and $\rho$ are the half chord of the deck and the air density respectively; both $b$ and $\rho$ are defined earlier. The aerodynamic derivatives are $L_{h}, L_{\alpha}, L_{\beta_{l}}, M_{h}, M_{\alpha}$ and $M_{\beta_{l}}$, and can be found in Graham 


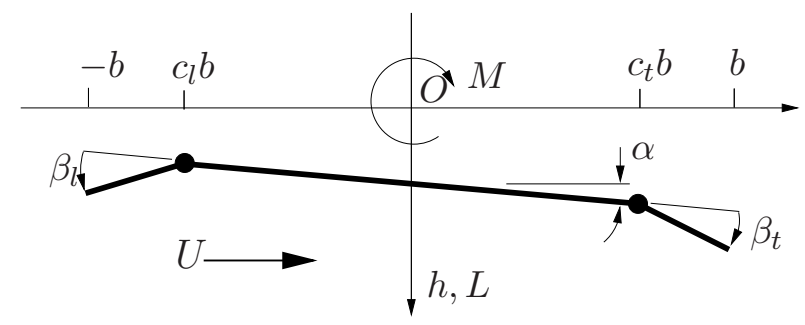

Figure 6: Kinematic model of the bridge deck. The origin of the inertial axis system is $O$. The wind velocity $U$ is assumed positive to the right (positive x-direction), the heave $h$ and lift force $L$ are assumed to be positive downwards, the moment $M$ is positive clockwise, as are the pitch and trailing-edge flap angles $\alpha$ and $\beta_{t}$ respectively. The leading-edge flap angle $\beta_{l}$ is positive anti-clockwise. The deck chord (including the flaps) is $2 b$. The leading- and trailing-edge flap chords are $\left(1+c_{l}\right) b$ and $\left(1-c_{t}\right) b$ respectively; note that $c_{l}$ is a negative quantity.

et al. (2011a,b). These formulae contain the irrational Theodorsen function Theodorsen (1934):

$$
C\left(k_{1}\right)=\frac{J_{1}\left(k_{1}\right)-i Y_{1}\left(k_{1}\right)}{\left(J_{1}\left(k_{1}\right)+Y_{0}\left(k_{1}\right)\right)-i\left(J_{0}\left(k_{1}\right)-Y_{1}\left(k_{1}\right)\right)},
$$

in which $J_{0}\left(k_{1}\right), J_{1}\left(k_{1}\right), Y_{0}\left(k_{1}\right)$ and $Y_{1}\left(k_{1}\right)$ are Bessel functions of the first and second kind respectively. To apply control-theoretic means we need a rational approximation of $C\left(k_{1}\right)$. By using a linear least squares approximation, this was derived in Graham et al. (2011a,b), with numerator and denominator coefficients as given in Table 2. Here $\hat{s}=\frac{s b}{U}$ is the reduced Laplace transform variable.

\subsection{Feedback Control System}

Figure 7 shows the bridge feedback control system. The uncontrolled open-loop system is described by the plant $P(s)$ that contains the structural dynamics and the non-circulatory part of the fluid mechanics. The Theodorsen function approximation $C(s)$ generates the circulatory flow; see Table 2. The unit-variance white-noise input $n(s)$ generates the atmospheric turbulence signal $u_{3}(s)$ using the filter $H(s)$, which is a stable and minimumphase spectral factor of the von Kármán turbulence spectrum approximation 


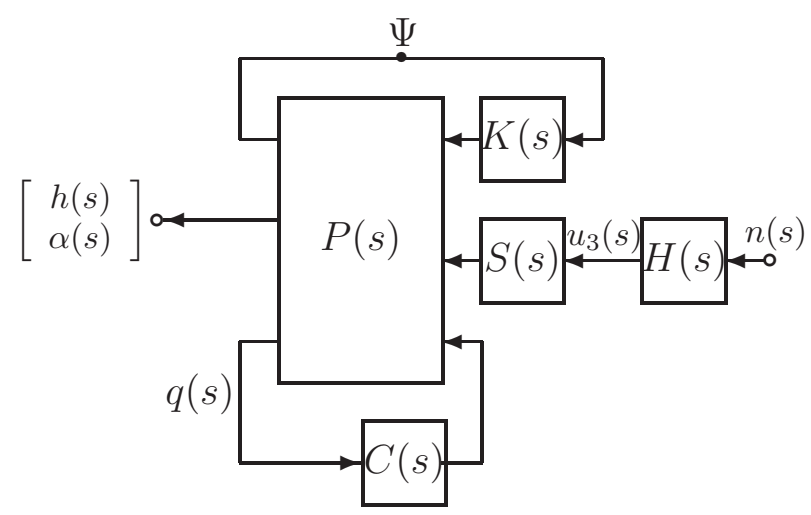

Figure 7: Block diagram of the aeroelastic control system. The dynamics of the bridge and non-circulatory fluid mechanics are represented by the plant $P(s)$, the Theodorsen circulation function approximation is given by $C(s)$ and the Sears function approximation (or the 3-dimensional correction given in (24)) is given by $S(s)$. The flap control system is given by $K(s)$ and the feedback signal by $\Psi$; the open-loop transfer function $Q(s)$ is obtained by 'looking into' the loop at $\Psi$ after it has been severed there. The wind gust vertical-velocity input is $w(s)$, with $H(s)$ the von Kármán turbulence filter and $n(s)$ a unit-variance white-noise process. The bridge deck's heave and pitch response are given by $h(s)$ and $\alpha(s)$ respectively. 


\begin{tabular}{||c||c||}
\hline numerator terms & denominator terms \\
\hline 0.99592 & 1 \\
\hline $57.01896 \hat{s}$ & $62.30441 \hat{s}$ \\
\hline $623.78848 \hat{s}^{2}$ & $807.78489 \hat{s}^{2}$ \\
\hline $1895.46328 \hat{s}^{3}$ & $3060.67868 \hat{s}^{3}$ \\
\hline $1523.24700 \hat{s}^{4}$ & $3033.76379 \hat{s}^{4}$ \\
\hline
\end{tabular}

Table 2: Numerator and denominator coefficients of a quartic approximation to the Theodorsen function.

Limebeer et al. (2011), which is given by

$$
\begin{aligned}
\tilde{H}\left(k_{1}\right)= & \sqrt{\frac{\sigma_{3}^{2} L_{y}}{U}} \frac{2.161402 k_{1}^{3}+8.960042 k_{1}^{2}}{k_{1}^{4}+6.151517 k_{1}^{3}+13.020580 k_{1}^{2}} . \\
& \frac{10.756042 k_{1}+3.7230314}{11.592094 k_{1}+3.723031},
\end{aligned}
$$

in terms of $k_{1}=\frac{s a L_{y}}{U}$, where a $=1.339$ is defined as in (4). The spanwise length scale of the vertical velocity is given by $L_{y}=0.05 \mathrm{~m}$, while $\sigma_{3}=0.065 U$ is the vertical rms turbulent velocity ( $U$ is the the freestream velocity). The measurement technique used to determine each of these parameters is mentioned at the end of Section 2. The poles and zeros of $\tilde{H}\left(k_{1}\right)$ are given in Table 3. The filter given in (31), when expressed in terms of the usual Laplace transform variable, appears as the filter $H(s)$ in Figure 7. A quartic approximation of the Sears function, given in Table 4, was used to generate the lift effects of atmospheric turbulence. For the purposes of the wind tunnel work the 3 -dimensional correction to $S(s)$, given in (24), is used and a unit reducedtime advance function $e^{s}$ must be appended to the quartic approximation Sears (1941). As with the approximation to the Theodorsen function, the quartic approximation to the Sears function is stable, minimum phase and with interlacing poles and zeros.

The controller

$$
K(s)=\left[\begin{array}{cc}
K_{l}(s) & 0 \\
0 & K_{t}(s)
\end{array}\right]
$$




\begin{tabular}{||c||c||}
\hline poles & zeros \\
\hline-2.84660037 & -2.32140736 \\
\hline-1.29484196 & -1.21169745 \\
\hline-1.01007454 & -0.61237243 \\
\hline-1.0 & - \\
\hline
\end{tabular}

Table 3: Poles and zeros of the stable and minimum-phase spectral factor of the von Kármán turbulence spectrum approximation.

\begin{tabular}{|c||c||}
\hline numerator terms & denominator terms \\
\hline 0.98820 & 1 \\
\hline $13.22326 \hat{s}$ & $18.10115 \hat{s}$ \\
\hline $28.55329 \hat{s}^{2}$ & $66.36398 \hat{s}^{2}$ \\
\hline $12.95482 \hat{s}^{3}$ & $60.356916 \hat{s}^{3}$ \\
\hline $0.72792 \hat{s}^{4}$ & $10.98343 \hat{s}^{4}$ \\
\hline
\end{tabular}

Table 4: Numerator and denominator coefficients of the quartic approximation to the Sears function.

generates leading- and trailing-edge flap angles.

The generalized state-space model of the system is

$$
\begin{aligned}
E \dot{x} & =A x+B u \\
z & =C x,
\end{aligned}
$$

which is an assembly of equations (26), (27), (28), (29) and the rational approximation $C(s), S(s)$ and $H(s)$. The control input $u(t)=\left[\beta_{l}(t) \beta_{t}(t)\right]^{\prime}$ in (32) comprises the leading- and trailing-edge flap angles, while the system output in (33) is either the vertical velocity of flap hinge points, or the deck's position, depending on the controller being used.

Since the controlled inputs (in the model) are the flap angles rather than torques applied to massy flap bodies, the model given in (32) must generate the first and second derivatives of the flap angles internally, which was 
achieved in Graham et al. (2011a) using a generalized state-space system of the form

$$
\left[\begin{array}{ll}
0 & 0 \\
1 & 0
\end{array}\right]\left[\begin{array}{l}
\dot{x}_{1} \\
\dot{x}_{2}
\end{array}\right]=\left[\begin{array}{ll}
1 & 0 \\
0 & 1
\end{array}\right]\left[\begin{array}{l}
x_{1} \\
x_{2}
\end{array}\right]+\left[\begin{array}{c}
-1 \\
0
\end{array}\right] \beta
$$

where $\beta$ denotes a flap angle and

$$
x_{1}=\beta ; \quad x_{2}=\dot{\beta} .
$$

It is clear that the matrix $E$ in (32) is singular. Thus we cannot easily design the buffeting controller using standard theory, and so the infinite-frequency modes were removed using a standard singular perturbation argument. The root loci in Figure 8, before and after this substitution are essentially identical. The singular perturbation made no identifiable difference to the Nyquist diagrams for the leading- and trailing-edge flap control loops either. These plots have been omitted.

It is determined from Figure 8 that the theoretical critical flutter speed is $20 \mathrm{~m} / \mathrm{s}$, while its critical torsional divergence speed is $24 \mathrm{~m} / \mathrm{s}$. The design aim is to increase the theoretical critical flutter speed to $24 \mathrm{~m} / \mathrm{s}$ (the critical torsional divergence speed), which represents a $20 \%$ improvement, and because velocity feedback cannot improve critical torsional divergence speed Graham et al. (2011a). From Section II we know that the real critical flutter speed of the bridge deck is $17.5 \mathrm{~m} / \mathrm{s}$; this $2.5 \mathrm{~m} / \mathrm{s}$ discrepancy is caused, Dyrbye and Hansen (1995), at least in part, by the fact that the bridge deck is not a thin plate; see Table 8.1 in Dyrbye and Hansen (1995). We expect that the controller designed from the model should also achieve a $20 \%$ improvement to $17.5 \mathrm{~m} / \mathrm{s}$, for the wind tunnel critical flutter speed. The flutter control system could achieve a larger increase in the critical wind speed if there was a larger gap between the critical flutter speed and the critical torsional divergence speed.

We consider control systems that use position feedback in which the controller $K(s)$ in Fig. 7 has both pitch angle and heave, or pitch angle only as input, and leading- and/or trailing-edge flap angles as output.

An alternative passive control system is the mechanical feedback control system shown in Figure 9, where deck motion is used to control the bridge flaps. The deck heave and pitch angle, and the leading- and trailing-edge flap angles are given by $h, \alpha, \beta_{l}$ and $\beta_{t}$ respectively. As seen in Figure 9 the 


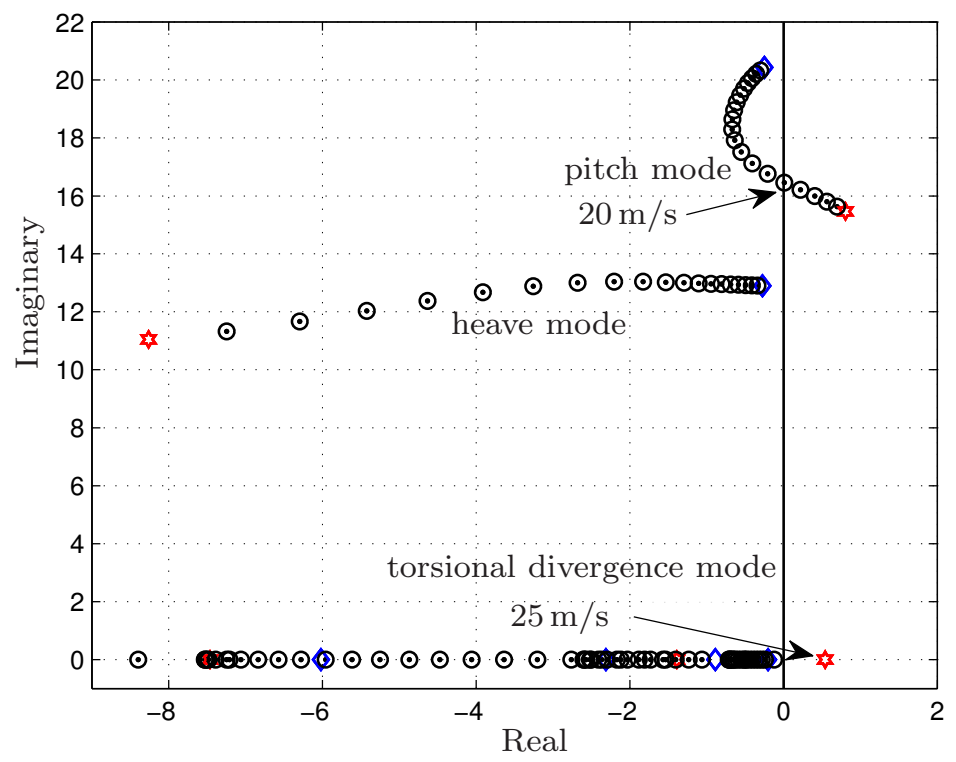

Figure 8: Root-loci of the uncontrolled bridge section. The wind speed is swept from $5 \mathrm{~m} / \mathrm{s}$ to $25 \mathrm{~m} / \mathrm{s}$, with the low-speed end of the root loci marked with (blue) diamonds and the high-speed ends marked with (red) hexagons. The root loci of uncontrolled system (32) - (33) with a singular $E$ are marked with black points, while the root-loci corresponding to the reduced order model are marked with black circles. The (flutter) pitch mode goes unstable at approximately $20 \mathrm{~m} / \mathrm{s}$, while the torsional divergence (zero frequency) mode goes unstable at approximately $24 \mathrm{~m} / \mathrm{s}$.

control system is made up of several components including two springs with spring constant $K_{s}$, two pinions with radius of $r$ that are mounted on the bridge deck and attached to the flaps and two compensation networks. The pinions are then driven by racks that are attached to the passive mechanical networks with admittances $Y_{l}(s)$ and $Y_{t}(s)$. The other end of the rack is connected to the springs $K_{s}$, which are anchored in the inertial reference framework.

While this is not shown in Figure 9 , the points $P_{t}^{\prime}$ and $P_{l}^{\prime}$ are ideally connected rigidly, through additional mechanical equipment, to the inertial reference frame thereby allowing the mechanical controllers to sense the vertical velocity of the flap hinge points. In a practical implementation an approximation will be required with these points anchored to deck and hanger components. As the deck moves, these mechanical admittances manipulate mechanically the flaps angles $\beta_{l}$ and $\beta_{t}$ using the rack-spring-and-pinion 


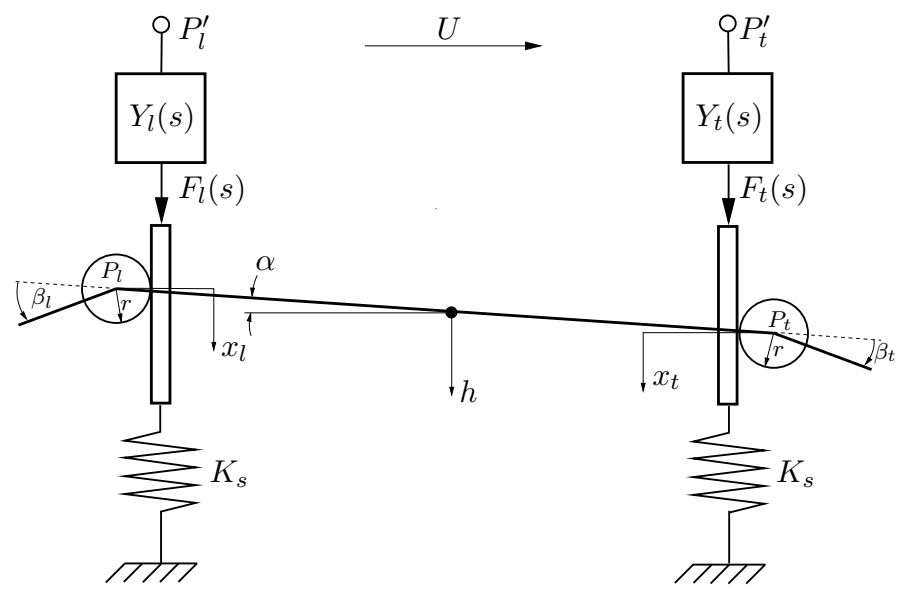

Figure 9: Mechanical network for flap feedback control.

connection to the flaps. If $Y_{l}(s)=Y_{t}(s)$ the control system in Figure 9 is insensitive to the wind direction. The proof is similar to that in Zhao et al. (2015), which has a similar controller that has the flap torques as control inputs.

In this work we use the mechanical network shown in Figure 10, which was originally proposed in Evangelou et al. (2007) in another context. The magnitude of its Bode plot has a bell shape which has high gain over the frequency range of interest, and low gain at low and high frequencies. These characteristics give zero DC gain allowing the flaps to operate around their zero positions, and suppress any high frequency noise. The transfer function of this network is

$$
Y_{d}(s)=\frac{K_{1} s}{s^{2}+\left(K_{1} / D_{1}\right) s+K_{1} / B_{1}}
$$

The series connection of the admittances $Y_{d}(s)$ and $K_{s}$ is given by

$$
\tilde{K}_{d}(s)=\frac{K_{s} Y_{d}(s)}{K_{s}+s Y_{d}(s)}
$$

Recall that the subscript $d$ can be either $l$ or $t$ depending on the wind direction.

The network through-variable associated with $\bar{K}_{d}(s)$ is the force $F_{d}(t)$, while the across-variable is the velocity of the flap hinge point $\dot{h}(t)+c_{d} b \dot{\alpha}(t)$. 


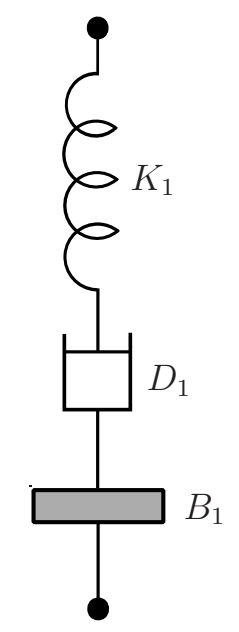

Figure 10: Realisation of a mechanical compensator for $Y_{l}(s)$ and $Y_{t}(s)$ in Figure 9, which comprise a spring $K_{1}$, a damper $D_{1}$ and an inerter $B_{1}$ in series.

Thus

$$
F_{d}(s)=\tilde{K}_{d}(s)\left(\dot{h}(s)+c_{d} b \dot{\alpha}(s)\right)
$$

The pinions rotate according as

$$
r \times \beta_{d}=\frac{F_{d}}{K_{s}}
$$

From (36), (37) and (38), we have

$$
K_{d}(s)=\frac{Y_{d}(s)}{r K_{s}+r s Y_{d}(s)},
$$

which maps $\dot{h}+c_{d} b \dot{\alpha}$ into the flap angles.

The control system design will focus on the optimisation of $K_{d}(s)$, which will be constrained to be passive and so:

$$
\int_{-\infty}^{T} F_{d}(t)\left(\dot{h}(t)+c_{d} b \dot{\alpha}(t)\right) d t \geq 0
$$

for all terminal times T, Smith (2002). 


\subsection{Robust Flutter and Buffet Controller Design}

The equipment listed in Section 2, such as computer, motors and so on were used to implement the position feedback control system and to simulate the mechanical system in Figure 9 in the wind tunnel experiments. The stepper motors and the low-pass filter introduce a significant phase delay that had to be included in the system model. The filter transfer function, implemented in Labview as a low-pass filter, is given by

$$
\begin{aligned}
& G_{f}(s)= \\
& \frac{3.209 e 08}{s^{5}+162.7 s^{4}+13230 s^{3}+665000 s^{2}+2.07 e 07 s+3.21 e 08} .
\end{aligned}
$$

We used a system identification experiment to obtain the transfer functions of the leading- and trailing-edge stepper motors $G_{l}$ and $G_{t}$ respectively:

$$
G_{l}(s)=\frac{s+0.4771}{s+0.2990}, \quad G_{t}(s)=\frac{s+78.3819}{s+76.0316} .
$$

With the above setting, the control system $K(s)$ in Figure 7 now consists of three parts:

$$
K(s)=G_{f}(s) G_{c}(s) G_{m}(s)
$$

where $G_{f}(s)$ is the above low-pass filter, $G_{c}(s)$ is the controller, and $G_{m}(s)$ is the above stepper motor. $K(s)$ can be SISO to drive one flap or MIMO to drive both flaps. Correspondingly $G_{c}(s)$ denotes either the trailing-edge controller, leading-edge controller or both, given in (39). Similarly, $G_{m}(s)$ denotes either the above trailing-edge motor $G_{t}(s)$ or leading-edge motor $G_{l}(s)$ or both. For the case of position feedback control system, we have similar formulation. The only difference is that the controller $G_{c}(s)$ now has the format as in (35).

For the flutter controller design, we require a passive network that stabilises the nominal closed loop and achieves the following closed-loop minimax objective:

$$
\min _{p}\left\{\max _{Q_{j}(s, p)}\left\|\left(I+Q_{j}(s, p)\right)^{-1}\right\|_{\infty}\right\} \quad j=1, \ldots n,
$$

which is used to optimise the closed-loop stability robustness to multiplicative perturbations Green and Limebeer (1995); the size (maximal gain) of the 
perturbation is less than the value of the index (42). The open-loop transfer functions $Q_{j}(s, p)=K(s) C_{j}\left(s E-A_{j}\right)^{-1} B_{j}$ corresponding to the wind speeds $U_{j} ; j=1, \ldots, n$ (from $4 \mathrm{~m} / \mathrm{s}$ to $24 \mathrm{~m} / \mathrm{s}$ in a step of $5 \mathrm{~m} / \mathrm{s}$ ) and are the multivariable transfer functions obtained by opening the feedback loop at $\Psi$ in Figure 7; $E, A_{j}, B_{j}, C_{j}$ are as in equations (32) and (33) corresponding to $U_{j}$. The Laplace transform variable is denoted $s$, with $\|\cdot\|_{\infty}$ the infinity norm Green and Limebeer (1995). The controller parameter set is given by $p$ and includes the compensator parameters, the pinion radius $r$ and spring constant $K_{s}$, which are optimised using the MATLAB ${ }^{\mathrm{TM}}$ sequential quadratic programming algorithm FMINCON.

To ensure that the optimisation problem is properly posed two constraints must be put in place that operate alongside (42). Firstly, to ensure stability, the closed-loop eigenvalues are constrained to have negative real parts. Secondly both the compensators $Y_{l}(s)$ and $Y_{t}(s)$ of the mechanical control system as in Fig. 9, and the controller $G_{c}(s)$ of the position feedback control systems are constrained to have the form of equation (35), which is positive real and can suppress DC gain and noise of the feedback signal.

The aim of the buffet control is to minimise the deck's buffet response using ideas from mixed $\mathcal{H}_{2} / \mathcal{H}_{\infty}$ optimal control theory; see Limebeer et al. (1994) and the references therein. The $\mathcal{H}_{2}$ optimal control serves to minimise the output (pitch and heave of bridge deck) variance under stochastic excitation, while $\mathcal{H}_{\infty}$ guarantees the buffet control still has certain robustness although less than the case of flutter control. The selected performance criterion is to minimise the power in the deck's heave and pitch responses due to wind turbulence. In this case the controller parameters are optimised to achieve the following closed-loop objective:

$$
\min _{p}\|H(s, p)\|_{2}
$$

in which $H(s, p)$ is the closed-loop transfer function for the system that maps the unit variance white noise $n(s)$ to $[h, \xi \alpha]^{T}$ in Figure $7 ;\|\cdot\|_{2}$ denotes the $\mathcal{H}_{2}$ norm. The constant $\xi$ is used to weight the relative importance of the heave and pitch responses; a value of $\xi=40$ is used throughout. To ensure that the optimisation problem of the buffet control design is well posed, we impose the same two constraints as the robust flutter controller design: closed-loop stability and network passivity. We will also impose a robust stability constraint: the value of (42) for the buffet controller is less than 3 times its value for the corresponding robust flutter controller. The 
exceptions are $\mathrm{BC} 1$ and $\mathrm{BC} 2$ in Table 6.

\subsection{Theoretical and Experiment Results with Position Feedback}

The optimal robust flutter and buffeting controllers found by FMINCON are summarised in Tables 5 and 6 respectively.

\begin{tabular}{|l|l|c|c|}
\hline No & Type and feedback & Flutter controllers & Value of (42) \\
\hline FC1 & LE: $\alpha$ & $-\frac{32.8185 s}{s^{2}+5.5575 s+182.667}$ & 1.3364 \\
\hline FC2 & TE: $\alpha$ & $-\frac{30.6758 s}{s^{2}+16.278 s+328.7332}$ & 2.0696 \\
\hline FC3 & LE, TE: $\alpha$ & $-\frac{70.9502 s}{s^{2}+130.2945 s+149.7662},-\frac{30.6882 s}{s^{2}+18.6618 s+310.4151}$ & 1.9621 \\
\hline FC4 & LE: $\alpha$, TE: $h$ & $-\frac{58.3121 s}{s^{2}+11.6757 s+81.5644},-\frac{79.126 s}{s^{2}+32.6885+157.1237}$ & 2.7729 \\
\hline
\end{tabular}

* The first controller is applied to the LE flap, while the second is applied to the TE flap.

Table 5: Flutter controllers with position feedback.

The root locus diagram in Figure 11 shows that the heave, pitch and torsional divergence modes are all well damped at a wind speed up to $U=$ $24 \mathrm{~m} / \mathrm{s}$ using the buffet control system BC4 in Table 6 . All the other controllers in Tables 5 and 6 improve the critical flutter speed from $20 \mathrm{~m} / \mathrm{s}$ to $24 \mathrm{~m} / \mathrm{s}$ (a 20\% improvement) in the theoretical model; the corresponding root-loci are omitted to save space. We expect that all these controllers will also achieve an experimental improvement of $20 \%$ in the critical flutter speed. Apart from controller $\mathrm{BC} 2$, the controllers work effectively for wind speeds up to $20.5 \mathrm{~m} / \mathrm{s}$ in the wind tunnel, which was our testing safety limit; $\mathrm{BC} 2$ achieves a critical flutter speed of just over $19.5 \mathrm{~m} / \mathrm{s}$. This slightly lower figure in the case of $\mathrm{BC} 2$ may be the result of reduced robustness, and/or because the larger operating flap angle causes more flow separation as compared with $\mathrm{FC} 1$ and $\mathrm{BC} 1$.

Figure 12 shows the effect of activating and deactivating the buffet control at a wind speed of $U=20.5 \mathrm{~m} / \mathrm{s}$ with controller BC4 as listed in Table 6 with an additional TE gain of 2. Our theoretical model is a flat-deck model, while 


\begin{tabular}{|l|l|c|c|}
\hline No & Type and feedback & Buffet controllers & Value of $(42)$ \\
\hline BC1 & LE: $\alpha$ & $-\frac{43.9878 s}{s^{2}+6.9839 s+185.6243}$ & 1.4434 \\
\hline BC2 & LE: $\alpha$ & $-\frac{151.1125 s}{s^{2}+16.8192 s+162.9963}$ & 4.5 \\
\hline BC3 & TE: $\alpha$ & $-\frac{32.0435 s}{s^{2}+7.85165 s+303.0837}$ & 6.2067 \\
\hline BC4 & LE, TE: $\alpha$ & $-\frac{79.7788 s}{s^{2}+14.628 s+68.7656},-\frac{69.3877 s}{s^{2}+11.6567 s+373.6953}$ & 5.8870 \\
\hline BC5 & LE: $\alpha$, TE: $h$ & $-\frac{110.0404 s}{s^{2}+20.1303 s+116.6551},-\frac{83.1464 s}{s^{2}+22.9503 s+89.3025}$ & 8.1480 \\
\hline
\end{tabular}

Table 6: Buffet controllers with position feedback.

the bridge deck in the wind tunnel is negatively cambered with significant thickness. In the wind tunnel the effect of the trailing-edge (TE) flap was found to be less than half of its theoretical values; this is discussed in more detail in Gouder et al. (2015). We have therefore compensated all the TE controllers using additional gains of between 2 and 3 times for all the TE controllers in the wind tunnel tests. The system is unstable in flutter at this wind speed when the flaps are locked to the deck; as soon as flutter controls are introduced the system stabilises and the flutter-related oscillations cease. The other controllers achieve a similar increase in the flutter speed.

The main purpose of this Section is to compare the theoretical and experimental results of the buffeting suppression performance of the controllers in Tables 5 and 6. These results are given in Figures 13 to 20, where the flutter and buffeting controllers appear in pairs according to the type and feedback. From these figures, we deduce that the experimental results achieved better performance for buffeting suppression than the theoretical predictions. As discussed in Section 3.3, this is because self-buffeting occurred in our wind tunnel tests; self-buffeting appears easier to control than aerodynamic buffeting due to incident turbulence. The former has more periodic content, while the latter is more random. From Figures 19 and 20 we can see that controllers FC4 and BC5 achieve a good suppression ratio for both pitch angle and heave, while the buffeting controller BC5 performs better than expected. 


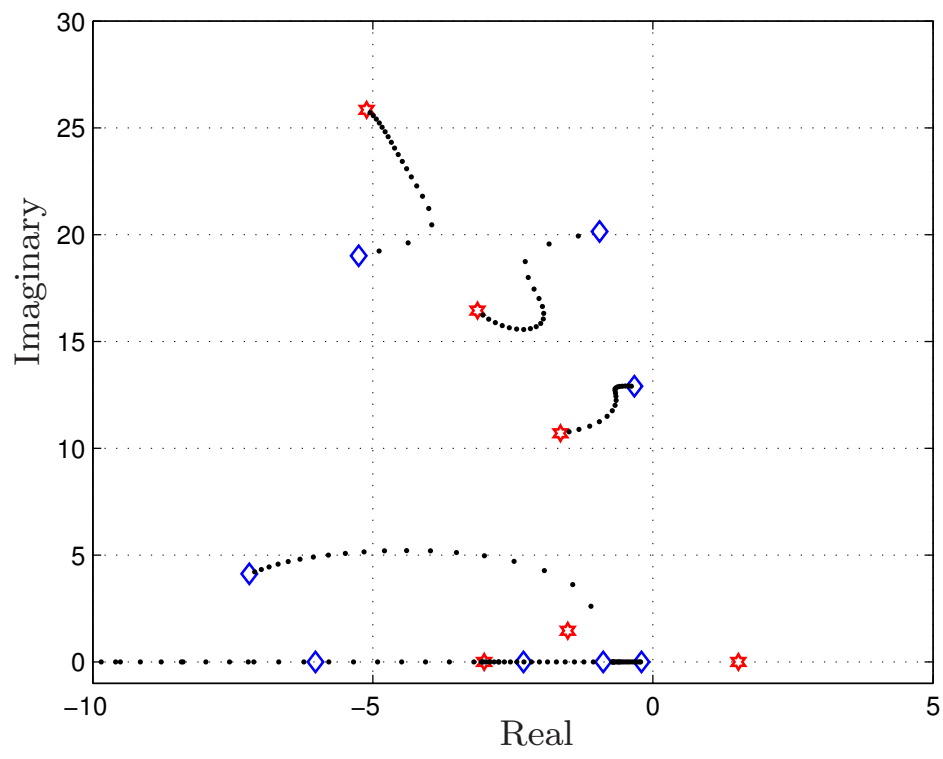

Figure 11: Root locus of the bridge section with controller BC4. The wind speed is swept from $5 \mathrm{~m} / \mathrm{s}$ to $25 \mathrm{~m} / \mathrm{s}$, with the low-speed end of the root loci marked with (blue) diamonds and the high-speed ends marked with (red) hexagons.

For all the other controllers, both the theoretical and experimental results, achieve a better suppression ratio for pitch angle as compared with heave. This is because the feedback signals are pitch angle with no heave content. In practice the major concern for buffet control is the pitch suppression.

All the buffet controllers have better pitch suppression performance than their pure-flutter counterparts (both theoretically and in practice), while their heave suppression performances vary. Figures 13 and 14 show the performance of buffet controllers $\mathrm{BC} 1$ and $\mathrm{BC} 2 ; \mathrm{BC} 2$ has much better buffeting suppression performance than $\mathrm{BC} 1$. However, due to its inferior stability margins, $\mathrm{BC} 2$ can only increase critical wind speed to just greater than $19.5 \mathrm{~m} / \mathrm{s}$. This demonstrates that if we lower, or remove, the increased flutter speed objective the leading-edge flap can achieve superior buffet suppression performance. 


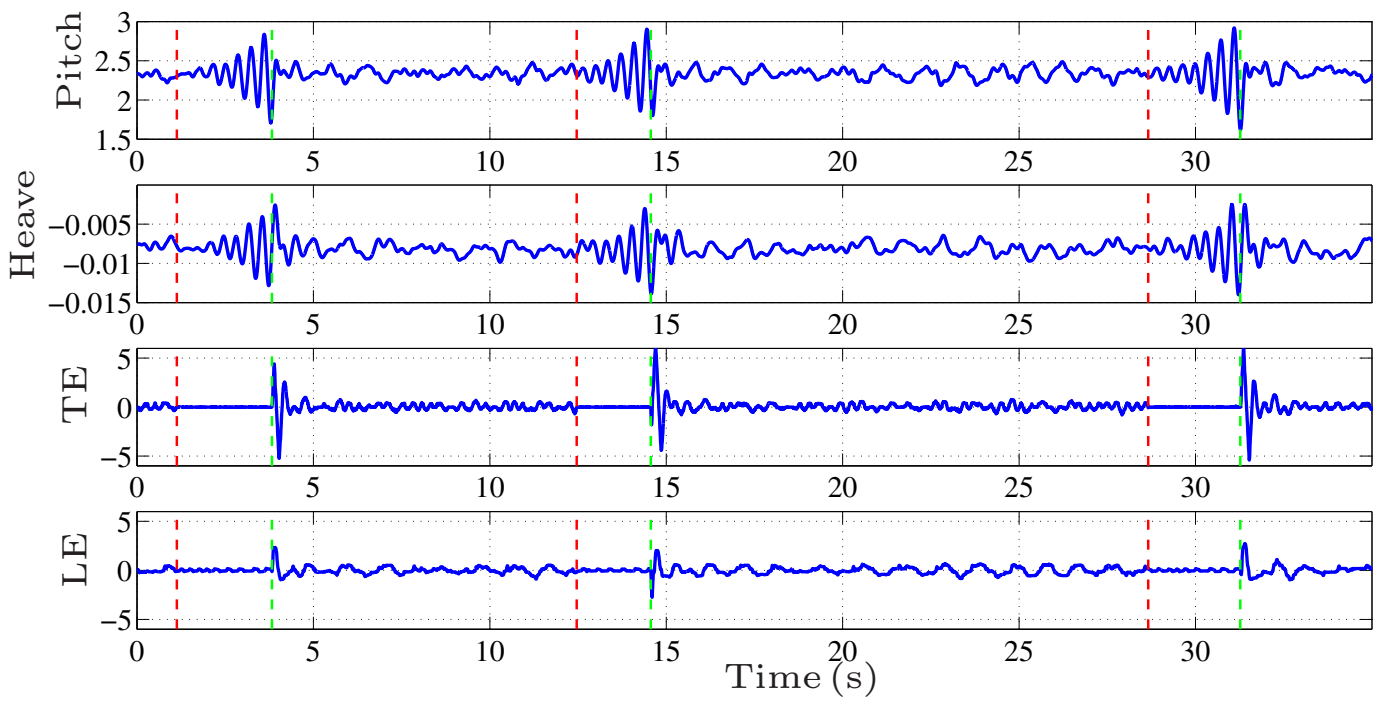

Figure 12: Stabilisation of flutter at a wind speed of $20.5 \mathrm{~m} / \mathrm{s}$ (the critical flutter speed is approximately $17.5 \mathrm{~m} / \mathrm{s}$ ) with buffet controller BC4 with TE gain 2 in Table 5 . The feedback loop is toggled open (green) and closed (red) at the vertical dashed lines. The plots show the deck pitch (in degrees) and heave (in meters), and the trailing (TE)- and leading (LE)-edge flap angles (in degrees). 

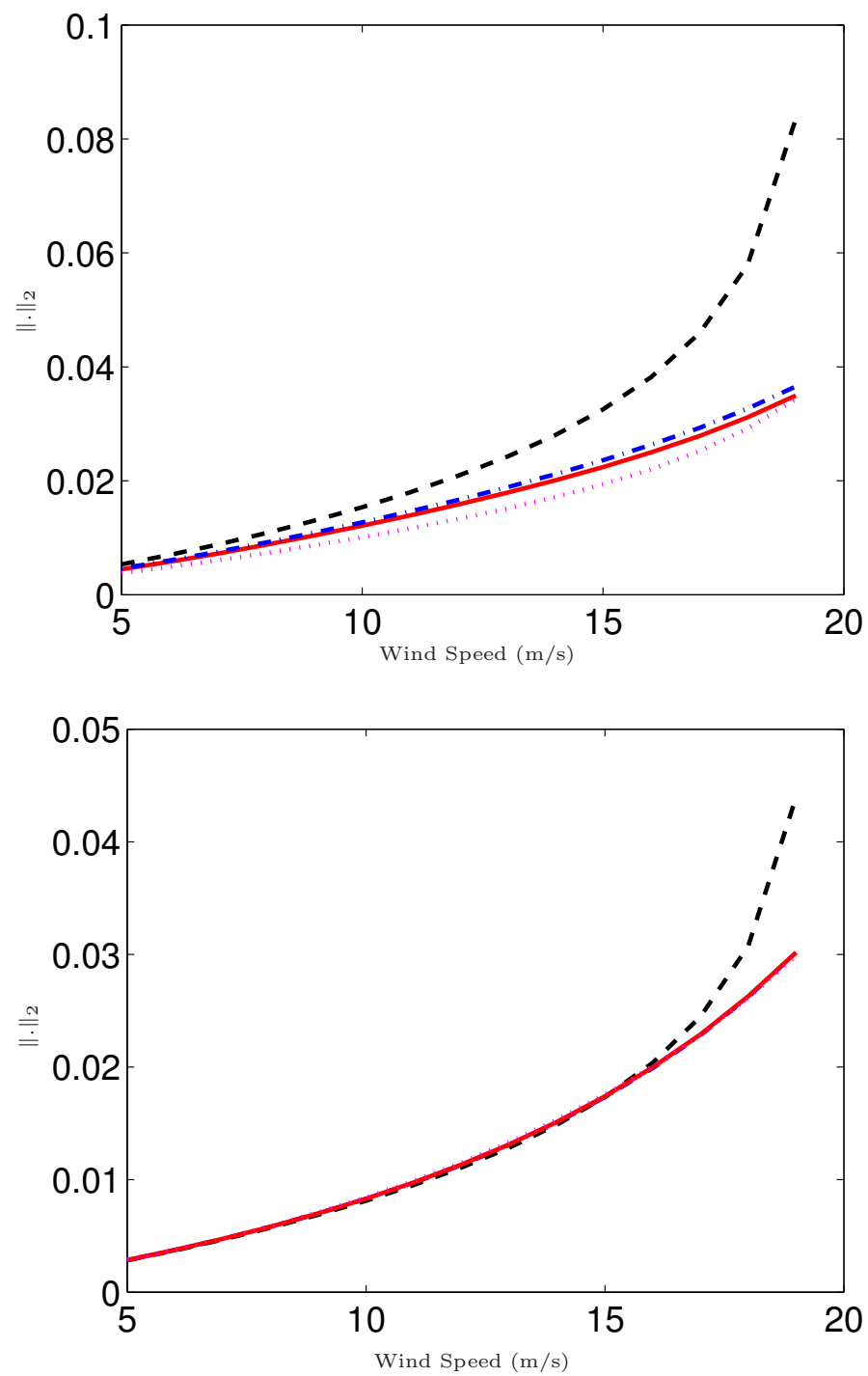

Figure 13: Theoretical feedback suppression of buffeting with control systems FC1, BC1 and $\mathrm{BC}$. The upper diagram shows the pitch angle response, while the lower diagram illustrates the heave response. The open-loop responses are the dashed (black) curves, the closed-loop responses achieved with flutter controller FC1 are the dot-dashed (blue) curves, the closed-loop responses achieved with buffet controller $\mathrm{BC} 1$ are the solid (red) curves curves, the closed-loop responses achieved with buffet controller $\mathrm{BC} 2$ are the dotted (magenta) curves. 

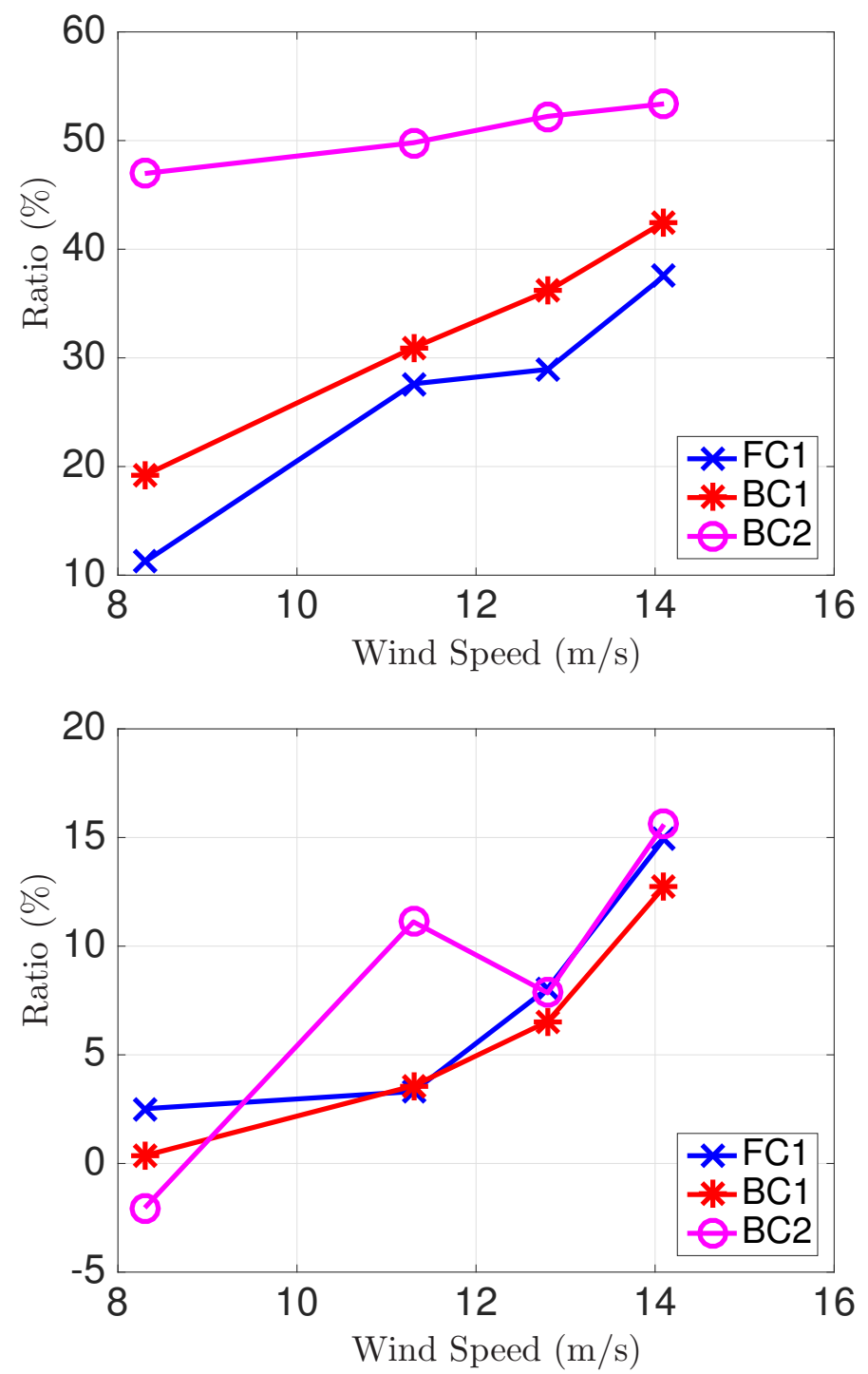

Figure 14: Experimental feedback suppression of buffeting with control systems FC1, $\mathrm{BC} 1$ and $\mathrm{BC} 2$ at hourly mean wind speeds: $8.3 \mathrm{~m} / \mathrm{s}, 11.3 \mathrm{~m} / \mathrm{s}, 12.8 \mathrm{~m} / \mathrm{s}$ and $14.1 \mathrm{~m} / \mathrm{s}$. The upper diagram shows the suppression ratio for pitch angle response, while the lower diagram illustrates the suppression ratio for heave response. The flutter control system FC1 is marked with blue x-marks, buffet control system BC1 is marked with red stars, $\mathrm{BC} 2$ is marked with magenta circles. 

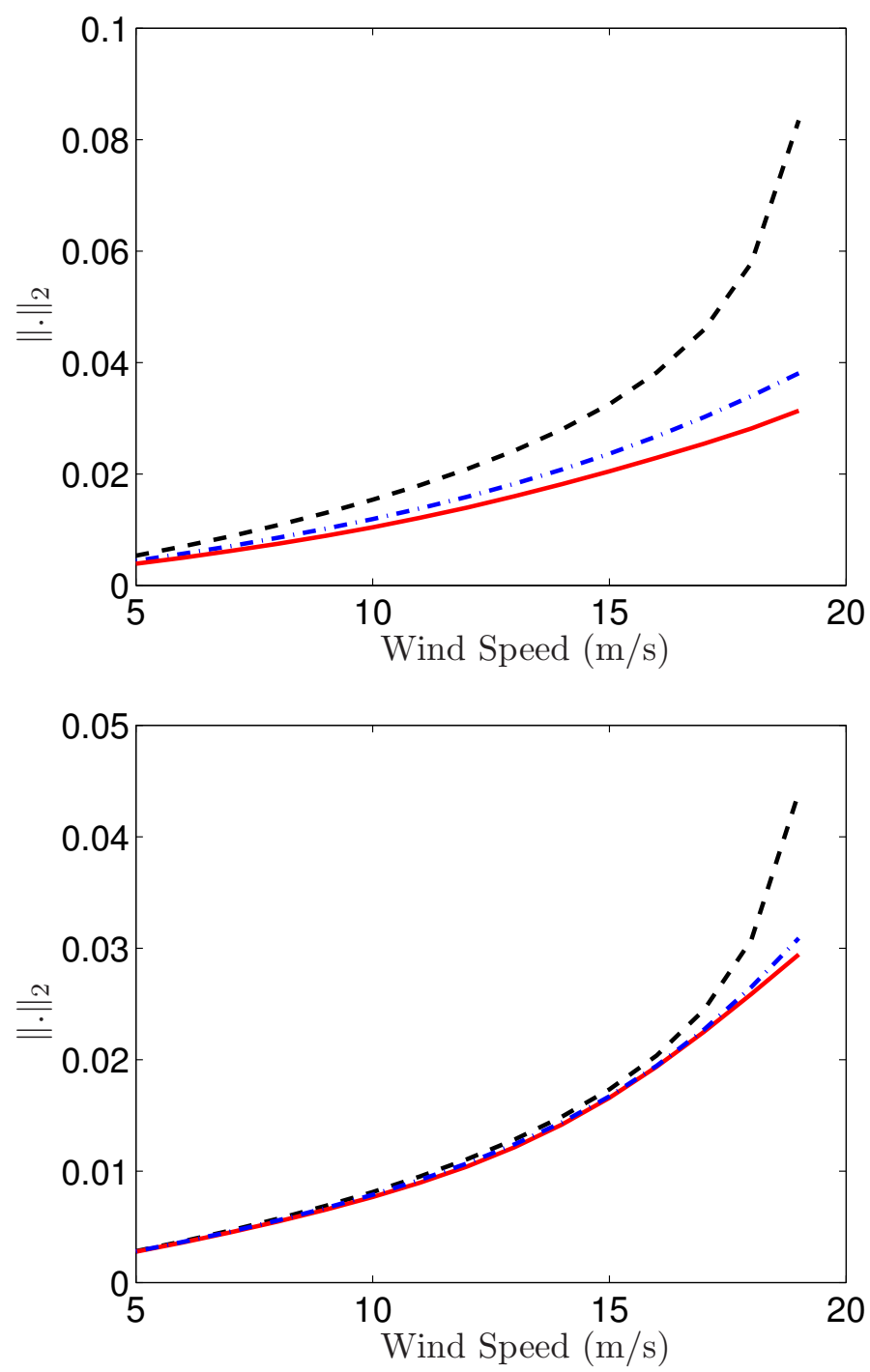

Figure 15: Theoretical feedback suppression of buffeting with control systems FC2 and BC3. The upper diagram shows the pitch angle response, while the lower diagram illustrates the heave response. The open-loop responses are the dashed (black) curves, the closed-loop responses achieved with flutter controller FC2 are the dot-dashed (blue) curves, the closed-loop responses achieved with buffet controller BC3 are the solid (red) curves. 

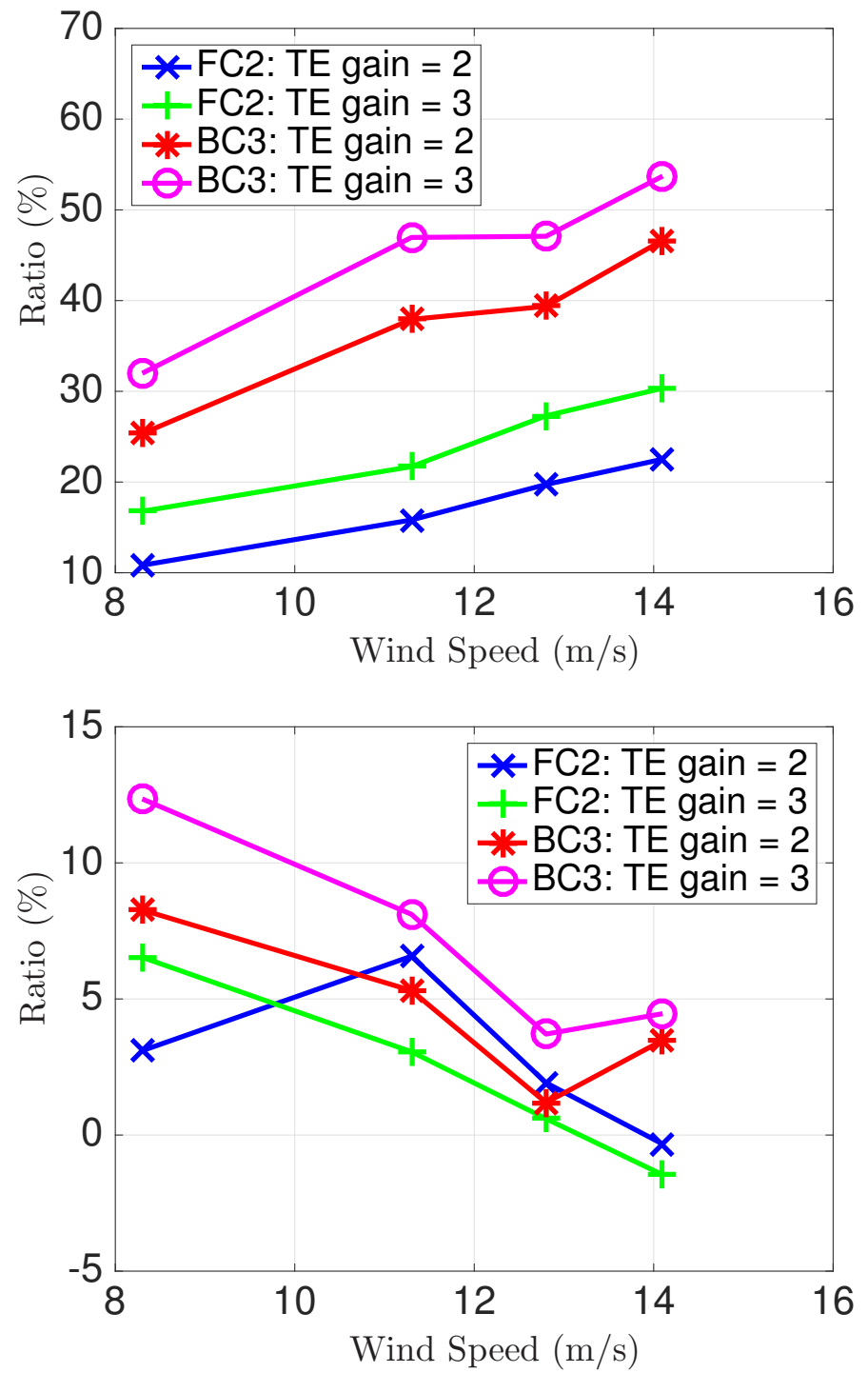

Figure 16: Experimental feedback suppression of buffeting with control systems FC2 and BC3 at hourly mean wind speeds: $8.3 \mathrm{~m} / \mathrm{s}, 11.3 \mathrm{~m} / \mathrm{s}, 12.8 \mathrm{~m} / \mathrm{s}$ and $14.1 \mathrm{~m} / \mathrm{s}$. The upper diagram shows the suppression ratio for pitch angle response, while the lower diagram illustrates the suppression ratio for heave response. The flutter control system FC2 with TE gain 2 is marked with blue $\mathrm{x}$-marks, $\mathrm{FC} 2$ with TE gain 3 is marked with green pluses, buffet control system BC3 with TE gain 2 is marked with red stars, BC3 with TE gain 3 is marked with magenta circles. 

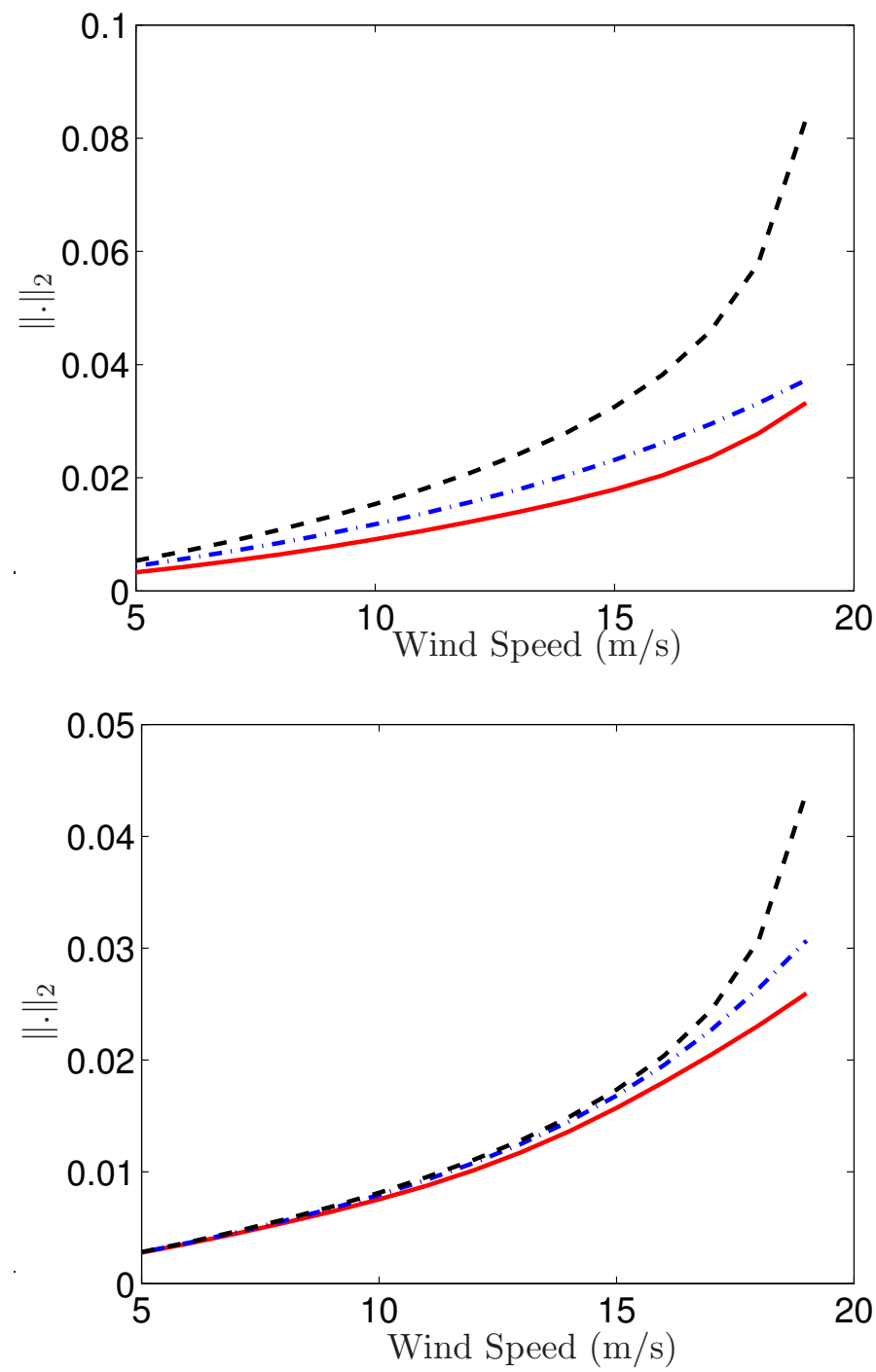

Figure 17: Theoretical feedback suppression of buffeting with control systems FC3 and BC4. The upper diagram shows the pitch angle response, while the lower diagram illustrates the heave response. The open-loop responses are the dashed (black) curves, the closed-loop responses achieved with flutter controller FC3 are the dot-dashed (blue) curves, the closed-loop responses achieved with buffet controller BC4 are the solid (red) curves. 

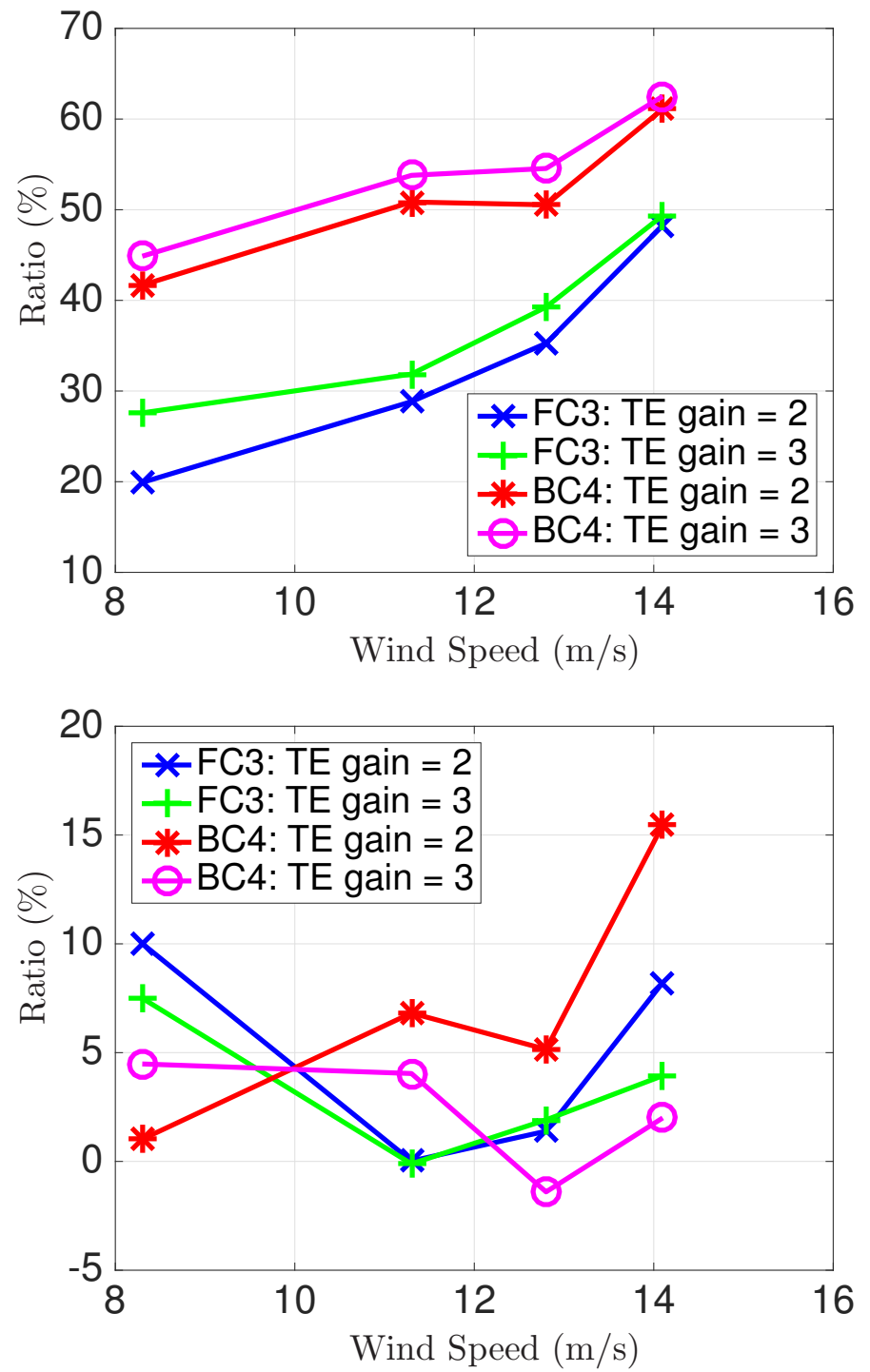

Figure 18: Experimental feedback suppression of buffeting with control systems FC3 and BC4 at hourly mean wind speeds: $8.3 \mathrm{~m} / \mathrm{s}, 11.3 \mathrm{~m} / \mathrm{s}, 12.8 \mathrm{~m} / \mathrm{s}$ and $14.1 \mathrm{~m} / \mathrm{s}$. The upper diagram shows the suppression ratio for pitch angle response, while the lower diagram illustrates the suppression ratio for heave response. The flutter control system FC3 with TE gain 2 is marked with blue $\mathrm{x}$-marks, FC3 with TE gain 3 is marked with green pluses, buffet control system BC4 with TE gain 2 is marked with red stars, BC4 with TE gain 3 is marked with magenta circles. 

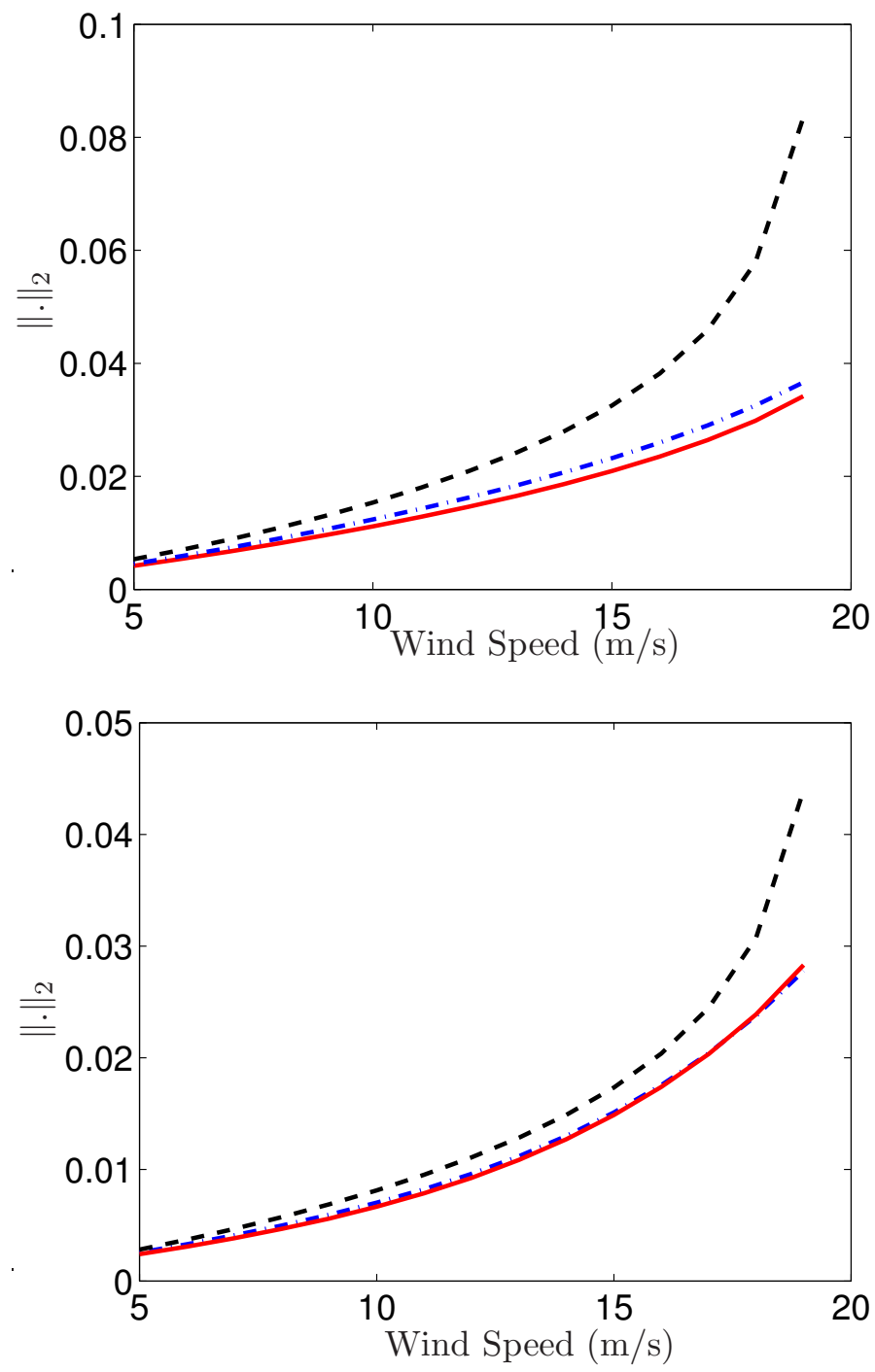

Figure 19: Theoretical feedback suppression of buffeting with control systems FC4 and BC5. The upper diagram shows the pitch angle response, while the lower diagram illustrates the heave response. The open-loop responses are the dashed (black) curves, the closed-loop responses achieved with flutter controller FC4 are the dot-dashed (blue) curves, the closed-loop responses achieved with buffet controller BC5 are the solid (red) curves. 

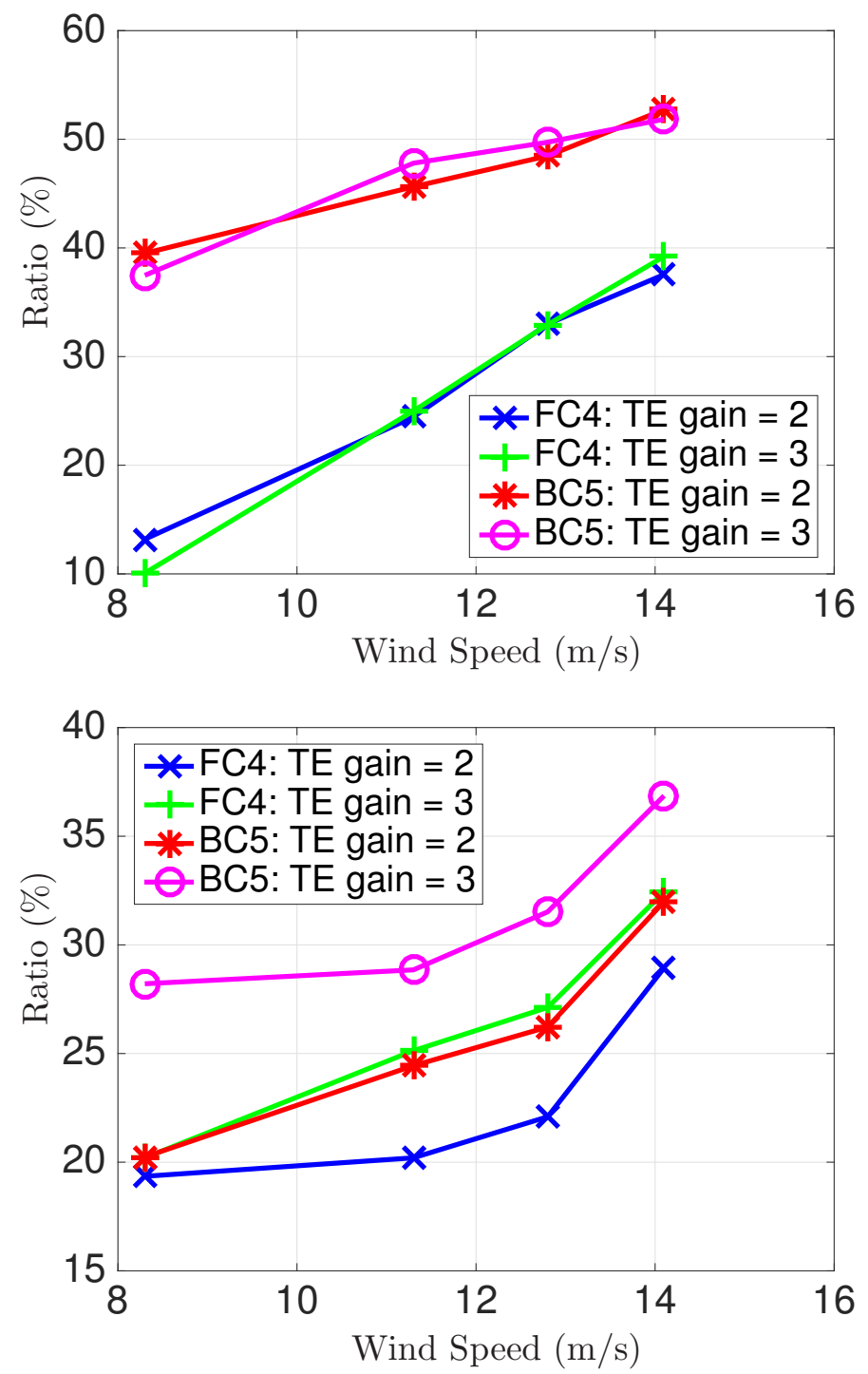

Figure 20: Experimental feedback suppression of buffeting with control systems FC4 and BC5 at hourly mean wind speeds: $8.3 \mathrm{~m} / \mathrm{s}, 11.3 \mathrm{~m} / \mathrm{s}, 12.8 \mathrm{~m} / \mathrm{s}$ and $14.1 \mathrm{~m} / \mathrm{s}$. The upper diagram shows the suppression ratio for pitch angle response, while the lower diagram illustrates the suppression ratio for heave response. The flutter control system FC4 with TE gain 2 is marked with blue $\mathrm{x}$-marks, FC4 with TE gain 3 is marked with green pluses, buffet control system BC5 with TE gain 2 is marked with red stars, BC5 with TE gain 3 is marked with magenta circles. 


\subsection{Theoretical and Experimental Results of Mechanical Control Systems}

This section considers the design of the mechanical control system in Figure 9 using the method alluded to in Section 4.3. The optimal robust mechanical flutter and buffet suppression controllers were found using FMIN$\mathrm{CON}$ and are summarised in Table 7. Only one mechanical buffet controller, $\mathrm{MBC} 1$, is considered that has a trailing-edge flap only. This restriction derives from the fact that buffet controllers with only leading-edge flaps, or control on both flaps cannot stabilise the bridge deck at wind speeds above $20.5 \mathrm{~m} / \mathrm{s}$ as was the case for BC2 in table VI. If we lower the critical flutter speed constraint below $20.5 \mathrm{~m} / \mathrm{s}$, one can obtain buffet controllers that have much better buffeting suppression performance than the corresponding flutter controllers. We have not explored this issue in any detail.

\begin{tabular}{|c|c|c|c|}
\hline No & Type & Mechanical controllers & Value of (42) \\
\hline MFC1 & $\mathrm{TE}$ & $\begin{array}{l}Y_{t}(s)=\frac{3.7572 s}{s^{2}+34.9751 s+820.5131} \\
K_{s}=35.4180 \mathrm{~N} / \mathrm{m} \\
r=0.0250 \mathrm{~m}\end{array}$ & 1.9024 \\
\hline MBC1 & $\mathrm{TE}$ & $\begin{array}{l}Y_{t}(s)=\frac{548.1711 s}{s^{2}=4177.68 s+90000} \\
K_{s}=35.0035 \mathrm{~N} / \mathrm{m} \\
r=0.0125 \mathrm{~m}\end{array}$ & 5.7000 \\
\hline $\mathrm{MFC} 2$ & LE & $\begin{array}{l}Y_{l}(s)=\frac{9.5516 s}{s^{2}+34.0549 s+782.0173}, \\
K_{s}=31.4946 \mathrm{~N} / \mathrm{m}, \\
r=0.0313 \mathrm{~m}\end{array}$ & 2.0410 \\
\hline MFC3 & LE, TE & $\begin{array}{l}Y_{d}(s)=\frac{4.9057 s}{s^{2}+77.7661 s+1551.3515} \\
K_{s}=27.4832 \mathrm{~N} / \mathrm{m} \\
r=0.0125 \mathrm{~m}\end{array}$ & 2.4894 \\
\hline
\end{tabular}

Table 7: Mechanical control systems.

The root locus diagram in Figure 21 shows that the heave, pitch and torsional divergence modes are all well damped at $U=24 \mathrm{~m} / \mathrm{s}$ by the flutter control system MFC3 as given in Table 7. All the other controllers in this table also improve the critical flutter speed from $20 \mathrm{~m} / \mathrm{s}$ to $24 \mathrm{~m} / \mathrm{s}$ (a $20 \%$ improvement) in the theoretical model. These controllers also improve the critical flutter speed to $20.5 \mathrm{~m} / \mathrm{s}$ (the highest test speed) in the wind tunnel. 


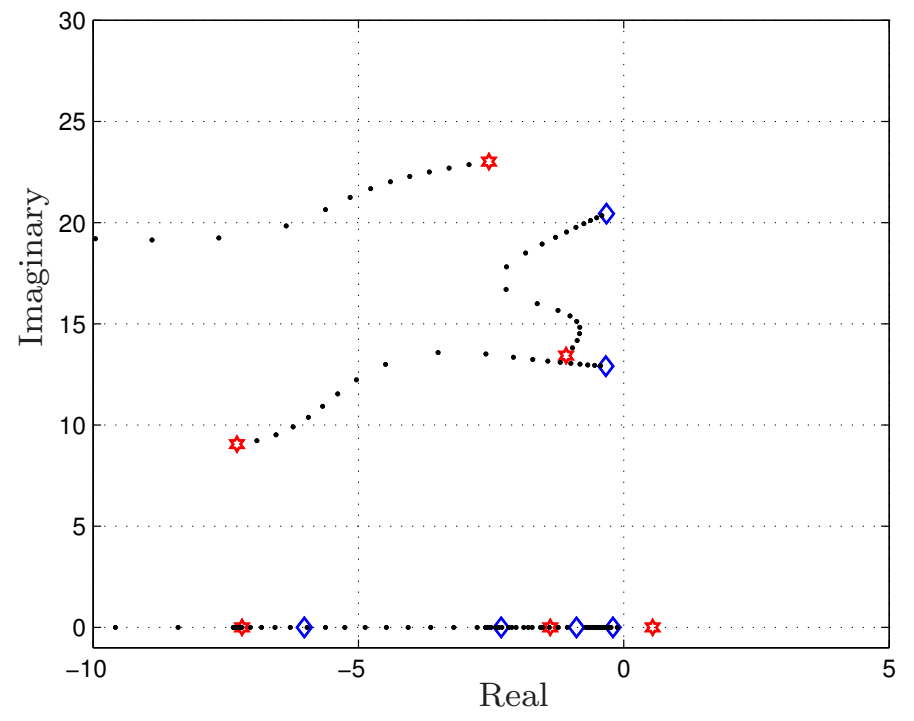

Figure 21: Root locus of the bridge section with flutter controller MFC3. The wind speed is swept from $5 \mathrm{~m} / \mathrm{s}$ to $25 \mathrm{~m} / \mathrm{s}$, with the low-speed end of the root loci marked with (blue) diamonds and the high-speed ends marked with (red) hexagons.

Figures 22 and 23 show the effect of activating and deactivating the flutter control system MFC3 at wind speeds of $U=18.5 \mathrm{~m} / \mathrm{s}$ and $U=20.5 \mathrm{~m} / \mathrm{s}$ respectively. It is self evident that in each case the systems are unstable in flutter when the flaps are locked to the deck. As soon as flutter controls are introduced the system stabilises and the flutter-related oscillations cease. It is also clear that it takes a much longer time for the controller to stabilise the system at $U=20.5 \mathrm{~m} / \mathrm{s}$ than at $U=18.5 \mathrm{~m} / \mathrm{s}$. The other controllers offer similar performances.

Figures 24 to 29 demonstrate the theoretical and experimental suppression of buffeting with the four control systems listed in Table 7 . These control systems all achieve a better suppression performance in the wind tunnel than the theory predicts; the reasons for this are analyzed in Section 4.4. As predicted by theory, all four controllers achieve a better experimental pitch suppression performance than heave suppression performance; this is probably because the heave velocity gain was set too small. As evidenced in Figures 24 and 25, the buffet control system MBC1 achieves a better pitch and heave suppression performance (theory and experiment) than the corresponding flutter control system MFC1. Also as predicted by theory, the 


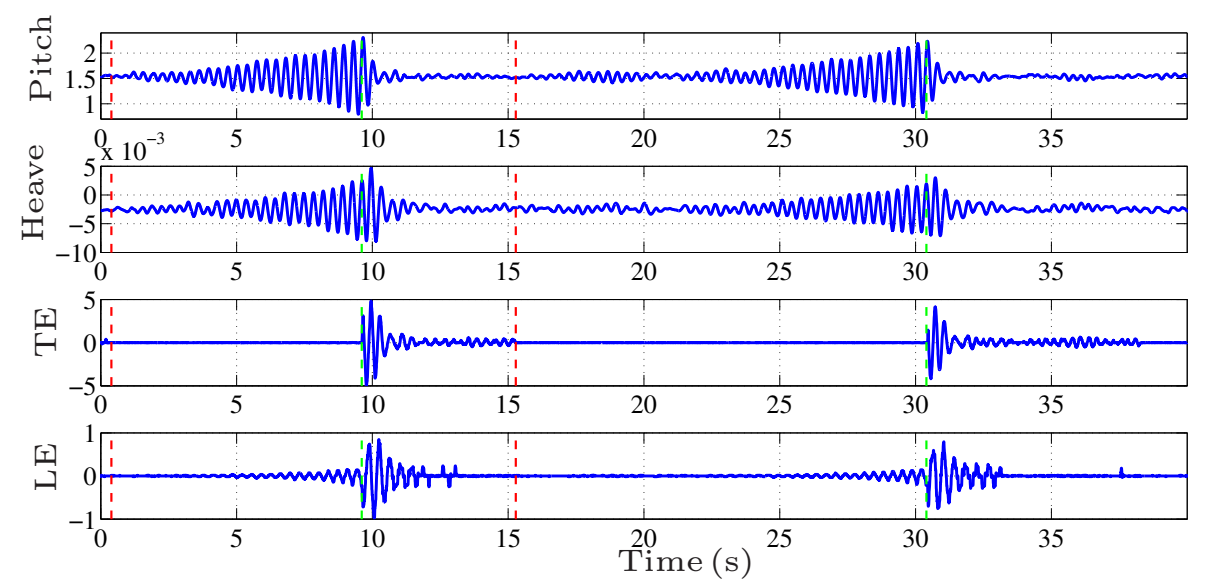

Figure 22: Stabilisation of flutter with control system MFC3 with TE gain 3 at a wind speed of $18.5 \mathrm{~m} / \mathrm{s}$ (the critical flutter speed is approximately $17.5 \mathrm{~m} / \mathrm{s}$ ). The feedback loop is toggled open (green) and closed (red) at the vertical dashed lines. The plots show the deck pitch (in degrees) and heave (in meters), and the trailing (TE)- and leading (LE)-edge flap angles (in degrees).

heave suppression by MFC2 and MFC3 becomes slightly worse in certain wind speed ranges in the wind tunnel.

\section{Conclusions}

This paper investigates two buffet-related problems for a model long-span suspension bridge deck mounted elastically in wind tunnels with two degrees of freedom. The first problem is the theoretical prediction of the dynamic response of a model bridge deck in grid-generated turbulence. This grid selected can generate a larger length-scale turbulent flow than the standard biplanar grid and thus causes a larger-amplitude bridge deck response. The measured spectra of the heave and pitch responses are compared with the corresponding spectra obtained from three-dimensional lifting surface theory. The measured and theoretical heave spectra agreed well, but the measured pitch spectrum was about 1.7 times bigger than its theoretical counterpart at the resonant peak. We believe that this difference is caused by self-buffet produced by flow separation. During experimental tests roll motions occurred, but they were small and thus could be neglected.

The second buffet-related problem is buffet suppression using controllable 


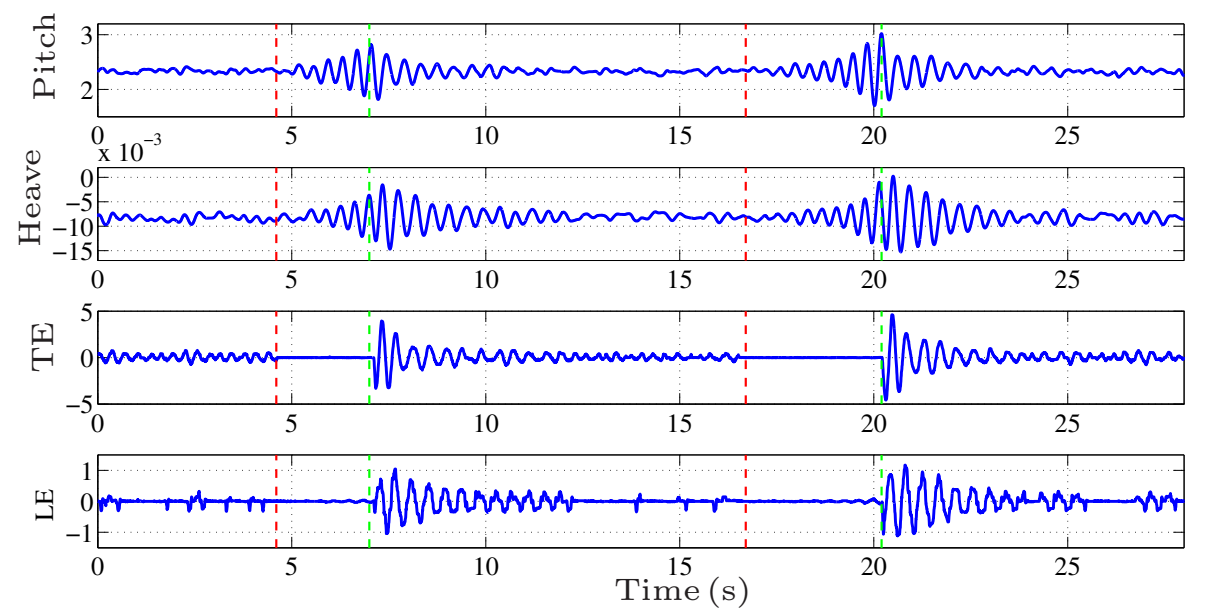

Figure 23: Stabilisation of flutter with control system MFC3 with TE gain 3 at a wind speed of $20.5 \mathrm{~m} / \mathrm{s}$ (the critical flutter speed is approximately $17.5 \mathrm{~m} / \mathrm{s}$ ). The feedback loop is toggled open (green) and closed (red) at the vertical dashed lines. The plots show the deck pitch (in degrees) and heave (in meters), and the trailing (TE)- and leading (LE)-edge flap angles (in degrees).

leading- and trailing-edge flaps that is investigated experimentally and theoretically. Two control systems are developed and tested. The first controller operates the flaps by sensing the leading-and/or trailing-edge deck position (pitch angle alone, or both pitch angle and heave). The second control system is a mechanism that operates the flaps by sensing the vertical velocity of the leading- and/or trailing-edge flap hinge points. Various flutter and buffet controllers of each type were developed and tested. Wind tunnel tests showed that all the controllers investigated effectively suppressed pitch buffet, with the buffet-related controllers achieving better pitch motion suppression than was achieved with controllers designed specifically to raise the critical flutter speed. Wind tunnel tests showed that these controllers achieved better buffet suppression (in pitch) than was predicted theoretically. This was probably a result of self-buffeting that occurred during testing, which is more amenable to control (more periodic components) than the aerodynamic buffeting caused by turbulence. Testing also indicated that almost all the controllers evaluated were insensitive to heave buffet. This was probably the result of small heave contributions to the control signals for the controllers tested; pitch buffet suppression is the key practice concern. Finally, it was 

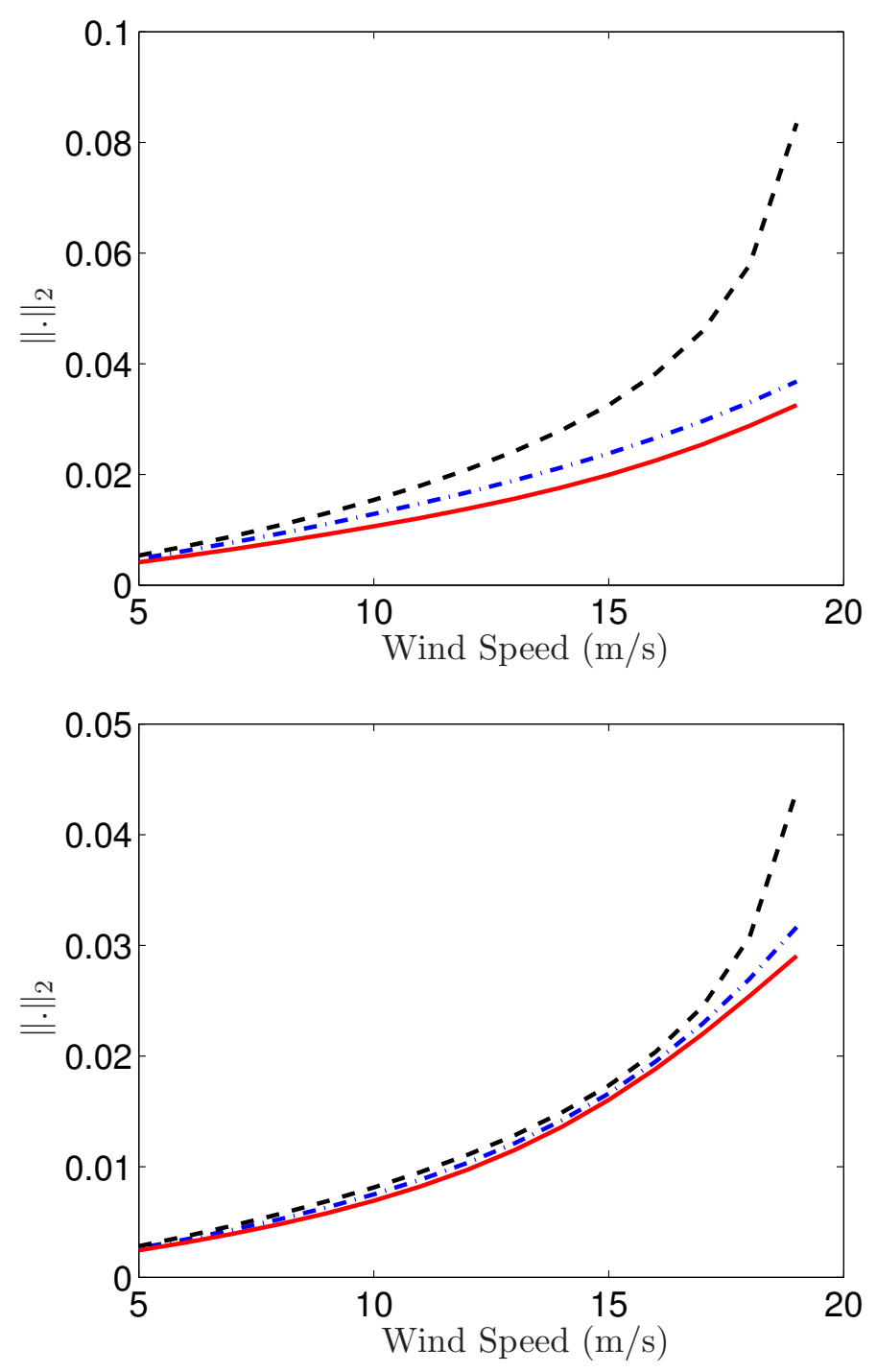

Figure 24: Theoretical feedback suppression of buffeting with control systems MFC1 and MBC1. The upper diagram shows the pitch angle response, while the lower diagram illustrates the heave response. The open-loop responses are the dashed (black) curves, the closed-loop responses achieved with flutter controller MFC1 are the dot-dashed (blue) curves, the closed-loop responses achieved with buffet controller MBC1 are the solid (red) curves.

experimentally verified that it is possible to effectively suppress buffeting, while simultaneously increasing the critical flutter speed of long-span bridge 

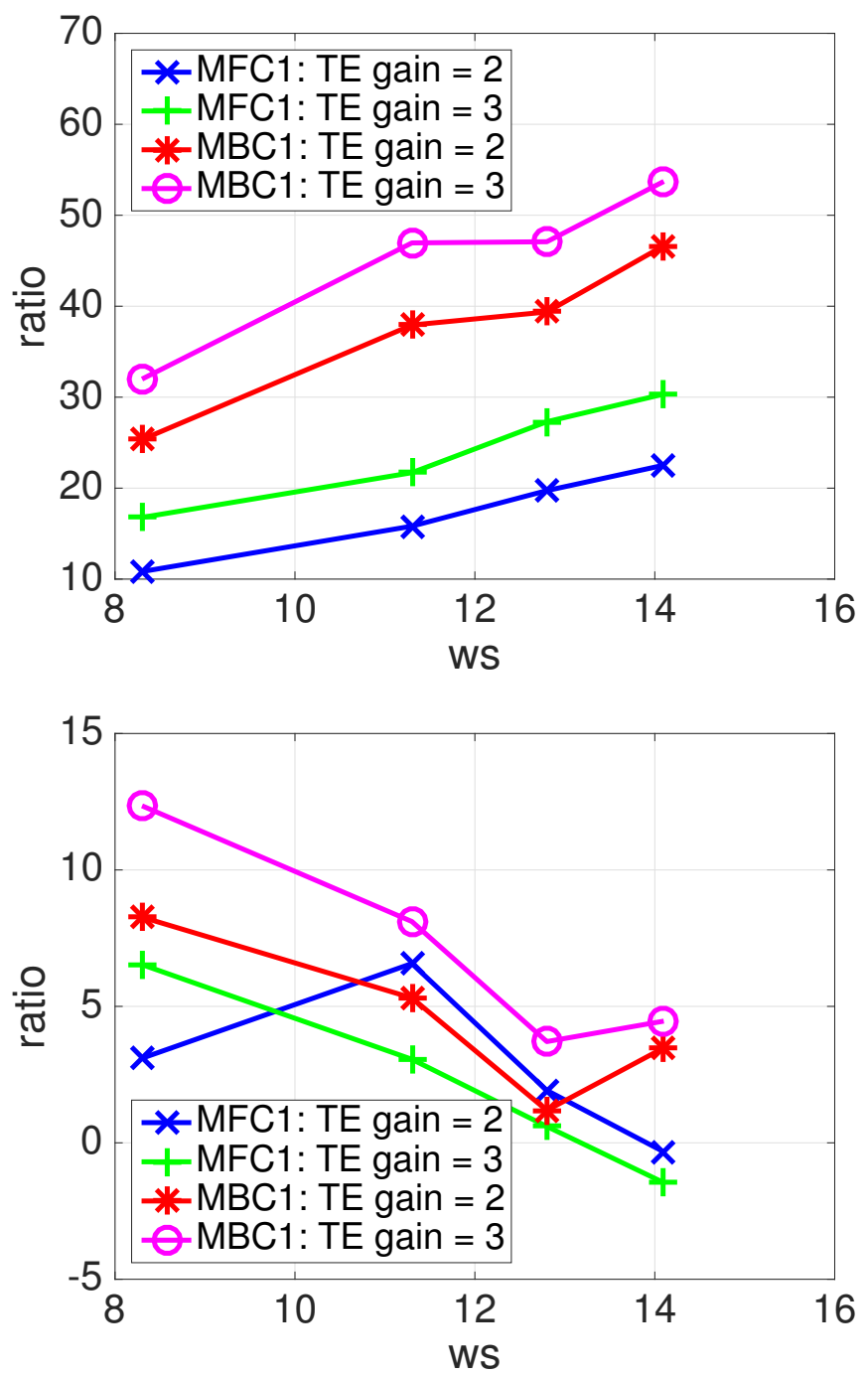

Figure 25: Experimental feedback suppression of buffeting with control systems MFC1 and MBC1 at hourly mean wind speeds: $8.3 \mathrm{~m} / \mathrm{s}, 11.3 \mathrm{~m} / \mathrm{s}, 12.8 \mathrm{~m} / \mathrm{s}$ and $14.1 \mathrm{~m} / \mathrm{s}$. The upper diagram shows the suppression ratio for pitch angle response, while the lower diagram illustrates the suppression ratio for heave response. The flutter control system MFC1 with TE gain 2 is marked with blue $\mathrm{x}$-marks, MFC1 with TE gain 3 is marked with green pluses, buffet control system MBC1 with TE gain 2 is marked with red stars, MBC1 with TE gain 3 is marked with magenta circles. 

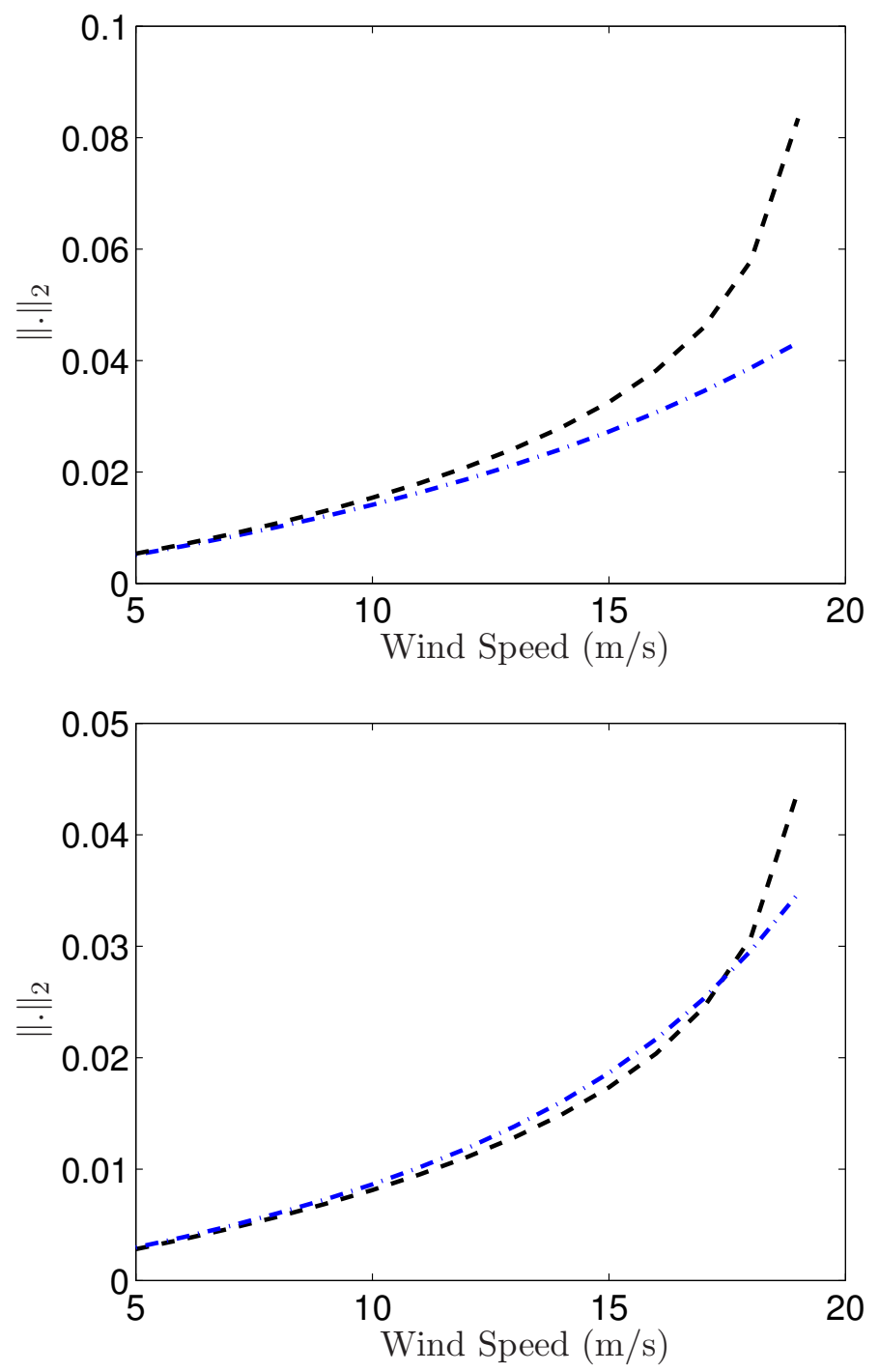

Figure 26: Theoretical feedback suppression of buffeting with control systems MFC2. The upper diagram shows the pitch angle response, while the lower diagram illustrates the heave response. The open-loop responses are the dashed (black) curves, the closed-loop responses achieved with flutter controller MFC2 are the dot-dashed (blue) curves.

decks. 

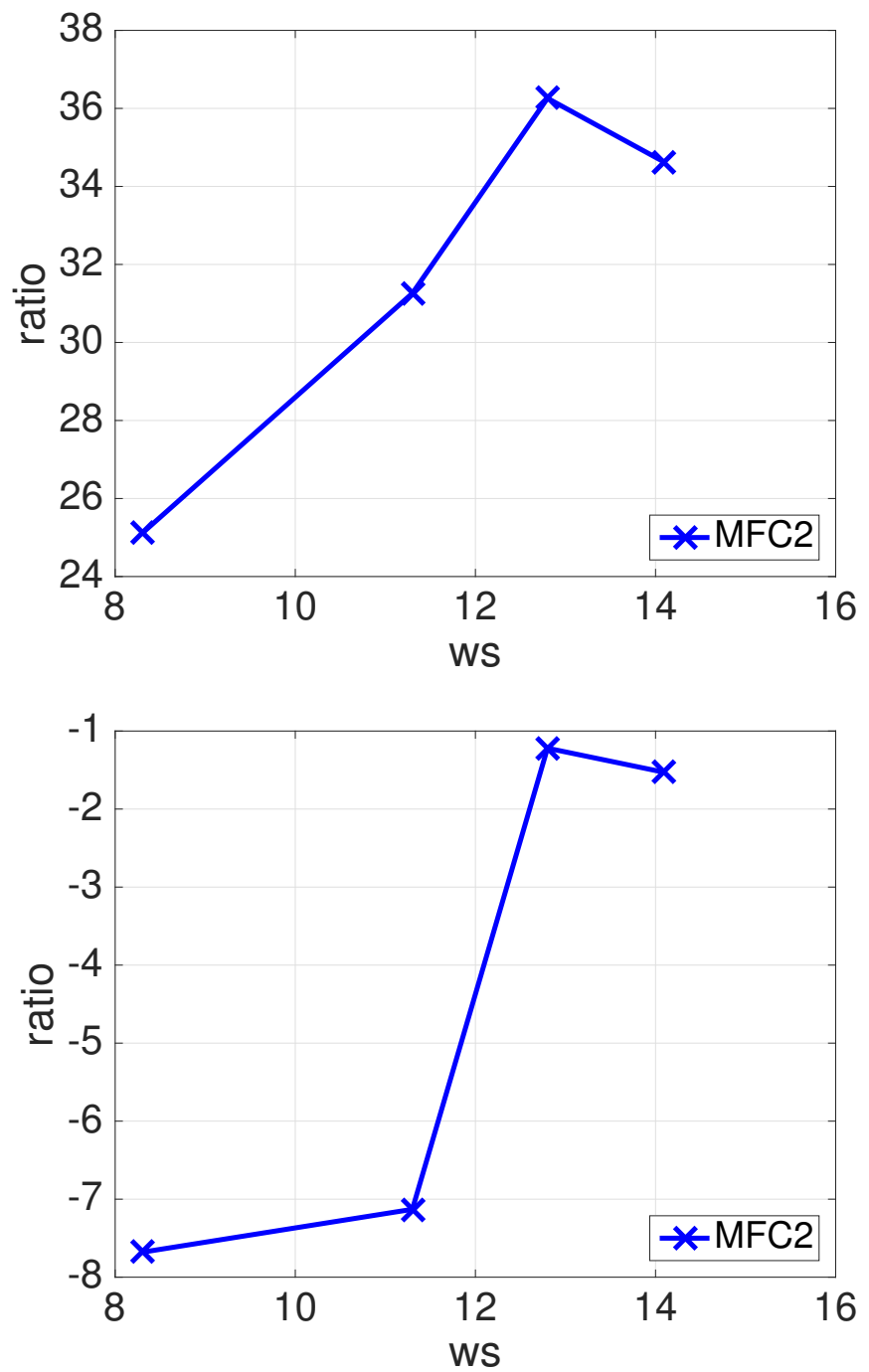

Figure 27: Experimental feedback suppression of buffeting with control systems MFC2 at hourly mean wind speeds: $8.3 \mathrm{~m} / \mathrm{s}, 11.3 \mathrm{~m} / \mathrm{s}, 12.8 \mathrm{~m} / \mathrm{s}$ and $14.1 \mathrm{~m} / \mathrm{s}$. The upper diagram shows the suppression ratio for pitch angle response, while the lower diagram illustrates the suppression ratio for heave response. The flutter control system MFC2 are marked with blue x-mark.

\section{Ackowledgements}

This work was supported by the Engineering and Physical Sciences Research Council (UK) and by BMT Fluid Mechanics Ltd. The additional wind 

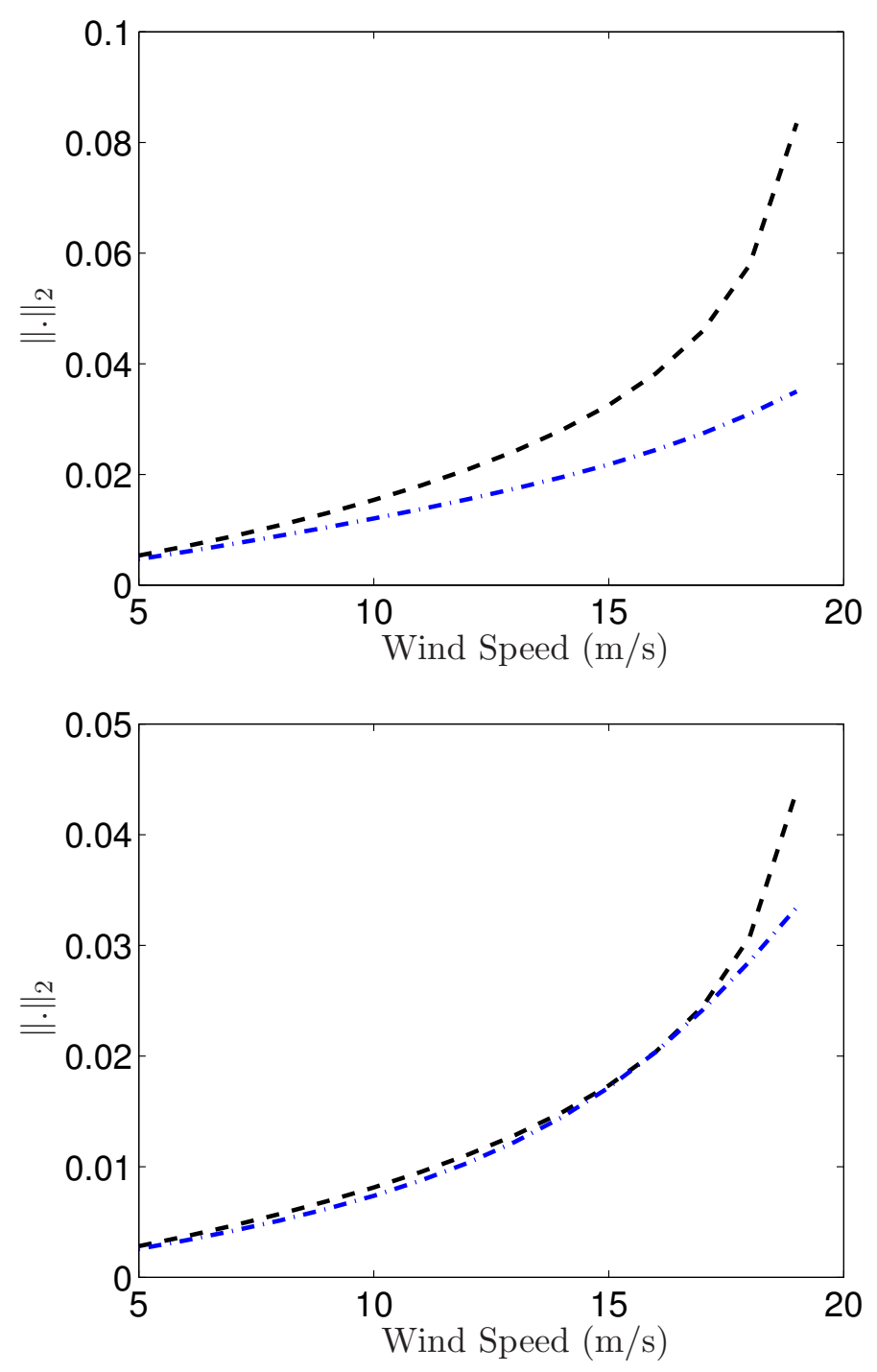

Figure 28: Theoretical feedback suppression of buffeting with control systems MFC3. The upper diagram shows the pitch angle response, while the lower diagram illustrates the heave response. The open-loop responses are the dashed (black) curves, the closed-loop responses achieved with flutter controller MFC3 are the dot-dashed (blue) curves.

tunnel time provided by BMT Fluid Mechanics and the support of their staff, Stefano Cammelli and Alvin Elliot is gratefully acknowledged. We would also like to thank professor Tom Wyatt for many insights and invaluable advice. 

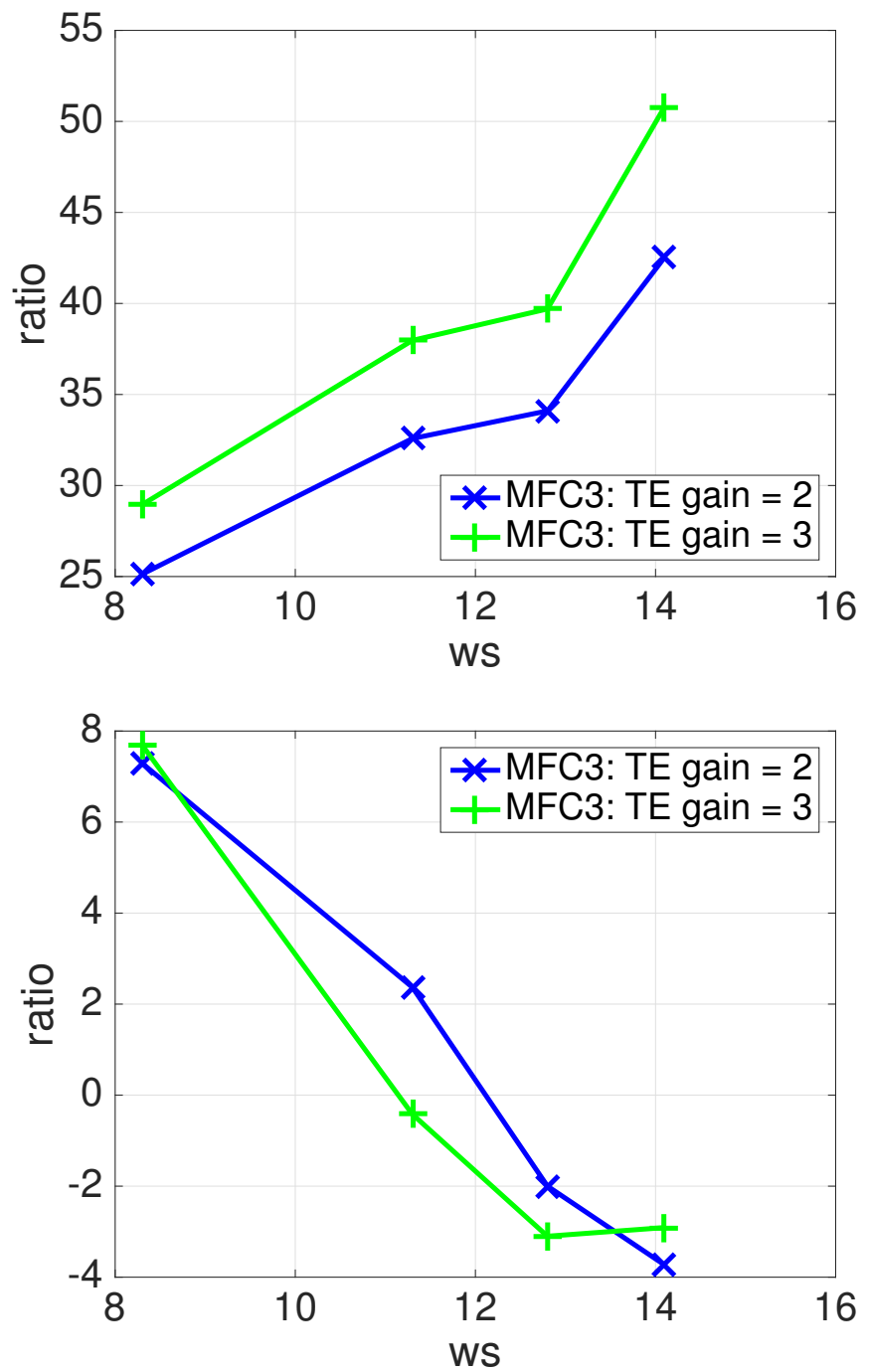

Figure 29: Experimental feedback suppression of buffeting with control systems MFC3 at hourly mean wind speeds: $8.3 \mathrm{~m} / \mathrm{s}, 11.3 \mathrm{~m} / \mathrm{s}, 12.8 \mathrm{~m} / \mathrm{s}$ and $14.1 \mathrm{~m} / \mathrm{s}$. The upper diagram shows the suppression ratio for pitch angle response, while the lower diagram illustrates the suppression ratio for heave response. The flutter control system MFC3 with TE gain 2 is marked with blue $\mathrm{x}$-marks, MFC3 with TE gain 3 is marked with green pluses.

\section{References}

Billah, K. Y., Scanlan, R. H., 1991. Resonance, tacoma narrows bridge failure, and undergraduate physics textbooks. American Journal of Physics 
59 (2), 118-124.

Borglund, D., Kuttenkeuler, J., 2002. Active wing flutter suppression using a trailing edge flap. Journal of Fluids and Structures 16 (3), 271 - 294.

Brown, D., 2001. Bridges: Three Thousand Years of Defying Nature. MBI Publishing Company, Saint Paul, USA.

Burnett, E., Atkinson, C., Sibbitt, B., Holm-Hansen, B., Nicolai, L., 2010. Ndof simulation model for flight control development with flight test correlation. In: AIAA Modeling and Simulation Technologies Conference.

Deaves, D., Harris, R., 1978. A mathematical model of the structure of strong winds. Construction Industry Research and Information Association: CIRIA Report No. 76.

Dyrbye, C., Hansen, S. O., 1995. Wind Loads on Structures, 1st Edition. John Wiley and Sons, Chichester.

Evangelou, S., Limebeer, D. J. N., Sharp, R. S., Smith, M. C., 2007. Steering compensators for high-performance motorcycles. ASME J. Applied Mechanics 74 (5), 332-346.

Frederick, M., Kerrigan, E. C., Graham, J. M. R., 2010. Gust alleviation using rapidly deployed trailing-edge flaps. Journal of Wind Engineering and Industrial Aerodynamics 98, 712723.

Gouder, K., Zhao, X., Limebeer, D., Graham, J., 2015. Experimental aerodynamic control of a section of long-span suspension bridge using leadingand trailing-edge control surfaces. IEEE Trans Control System Technology,Under Review.

Graham, J., 1970. Lifting surface theory for the problem of an arbitrarily yawed sinusoidal gust incident on a thin aerofoil in incompressible flow. Aero. Quarterly 21, 182-198.

Graham, J., Limebeer, D., Zhao, X., 2011a. Aeroelastic control of longspan suspension bridges. ASME J. Applied Mechanics 78 (4), 041018.1 - 041018.12. 
Graham, J. M. R., Limebeer, D. J. N., Zhao, X., 28 August to 2 September 2011b. Aeroelastic moldelling of long-span suspension bridges. In: Proc. of the 18th IFAC World Congress. Proc. of the 18th IFAC World Congress, Milano, Italy, Milan, pp. 9212 - 9217.

Green, M., Limebeer, D. J. N., 1995. Linear Robust Control. Prentice Hall, Englewood Cliffs, New Jersey.

Hansen, H. I., Thoft-Christensen, P., 2001. Active flap control of long suspension bridges. Journal of Structural Control 8 (1), 33-82.

Jackson, R., Graham, J. M. R., Maull, D. J., 1973. The lift on a wing in a turbulent flow. Aeronautical Quarterly 24, 155-166.

Kaimal, J., Wyngaard, J., Izumi, Y., Cote, O., 1972. Spectral characteristics of surface layer turbulence. Quarterly Journal of the Royal Meteorological Society Journal of Wind Engineering and Industrial Aerodynamics 98, $563-589$.

Karpel, M., 1982. Design for active flutter suppression and gust alleviation using state-space aeroelastic modeling. Journal of Aircraft 19 (3), 221-227.

Larose, G. L., Livesey, F. M., 1997. Performance of streamlined bridge decks in relation to the aerodynamics of a flat plate. Journal of Wind Engineering and Industrial Aerodynamics 69 - 71, 851 - 860, proceedings of the 3rd International Colloqium on Bluff Body Aerodynamics and Applications.

Larose, G. L., Mann, J., 1998. Gust loading on streamlined bridge decks. Journal of Fluids and Structures 12 (5), 511-536.

Limebeer, D. J. N., Anderson, B. D. ., Hendel, B., 1994. A nash game approach to mixed $\mathcal{H}_{2} / \mathcal{H}_{\infty}$ control. IEEE Trans. Auto. Control 39 (1), 69-82.

Limebeer, D. J. N., Graham, J. M. R., Zhao, X., 2011. Buffet suppression in long-span suspension bridges. Annual Reviews in Control 35, 235-246.

Ostenfeld, K. H., Larsen, A., February 1992. Bridge engineering and aerodynamics, aerodynamics of large bridges. In: Proceedings of the First International Symposium on Aerodynamics of Large Bridges. Copenhagen, Denmark, pp. 19-21. 
Roger, K. L., Hodges, G. E., Felt, L., 1975. Flutter supprsuppress - a flight demonstration. J. Aircraft 12 (6).

Sears, W. R., 1941. Some aspects of non-stationary airfoil theory and its practical application. AIAA Journal Special Supplement: Centennial of powered flight 8 (3), 104-108.

Selberg, A., Hjorth-Hansen, E., 1976. The fate of flat-plate aerodynamics in the world of bridge decks. In: Proceedings of the Theodorsen Colloquium. Trondheim, pp. 101-113.

Sharma, A., Limebeer, D. J. N., 2012. Motorcycle suspension design using matrix inequalities and passivity constraints. Vehicle System Dynamics: International Journal of Vehicle Mechanics and Mobility 50, 377-393.

Smith, M. C., October 2002. Synthesis of mechanical networks: The inerter. IEEE Trans. Automatic Control 47 (10), 1648-1662.

Theodorsen, T., 1934. General theory of aerodynamic instability and the mechanisms of flutter. NACA Report, TR-496.

Triplett, W. E., 1972. A feasibility study of active wing/store flutter control. J. Aircraft 9 (6).

Triplett, W. E., Kappus, H. P. F., Landy, R. J., 1973. Active flutter control - an adaptable application to wing/store flutter. J. Aircraft 10 (11).

Wilde, K., Fujino, Y., 1996. Variable-gain control applied to aerodynamic control of bridge deck flutter. In: Proceedings of 35th Conference on Decision and Control, Kobe, Japan. Proceedings of 35th Conference on Decision and Control, Kobe, Japan, Kobe, Japan, pp. 682-687.

Zhao, X., Limebeer, D. J. N., Graham, J. M. R., 12 December to 15 December 2011. Flutter control of long-span suspension bridges. In: Proc. of the 50th IEEE Conference on Decision and Control. Proc. of the 18th IFAC World Congress, Milano, Italy, Orlando, FL, US, pp. 4195 - 4200.

Zhao, X., Limebeer, D. J. N., Graham, J. R., Gouder, K., 2015. Aerodynamic control of a sectional suspended-span bridge model. Automatica.Under review. 